\title{
Anthrax Sampling and Decontamination: Technology Trade-Offs
}

\author{
Phillip N. Price, Kristina Hamachi, \\ Jennifer McWilliams, and Michael D. Sohn \\ Environmental Energy Technologies Division \\ Lawrence Berkeley National Laboratory \\ Berkeley CA 94720
}

September 12, 2008

This work was supported by the Office of Science, Office of High Energy Physics, Homeland Security under the U.S. Department of Energy under Contract No. DE-AC02-05CH1123. 


\section{Contents}

1 Executive Summary 3

1.1 How much sampling is needed to decide if a building is safe? . . . . . . . 3

1.1.1 Sampling Nomogram . . . . . . . . . . . . . . . . . . 4

1.2 How many characterization samples should be taken? . . . . . . . . . 7

1.3 What decontamination method should be used? . . . . . . . . . . . 7

1.4 Post-decontamination sampling . . . . . . . . . . . . . 8

1.5 What are rules of thumb for cost and effort? . . . . . . . . . . . . . 9

1.6 Conclusions . . . . . . . . . . . . . . . . . . . 10

2 Introduction $\quad 12$

3 How Clean is Safe? 14

3.1 Dose-Response Relationship for Anthrax . . . . . . . . . . . . . . . . . . 15

3.2 Relationship between Surface Concentration and Dose . . . . . . . . . . . 17

3.3 Setting a Safety Standard . . . . . . . . . . . . . . . . . 19

4 Spatial Distribution of Contamination $\quad 22$

4.1 Hart Building . . . . . . . . . . . . . . . . . . . . . . . 24

4.2 Other Capitol Hill locations . . . . . . . . . . . . . . . . . . 25

4.3 Brentwood . . . . . . . . . . . . . . . . 25

4.4 American Media, Inc. (AMI) Building . . . . . . . . . . . . . 26

4.5 Other Data on the Spatial and Temporal Spread of Contamination . . . . 28

4.6 Rough prediction of surface concentrations . . . . . . . . . . . . . . . 29

5 Sampling 35

5.1 Detection Technologies and Effectiveness . . . . . . . . . . . . 35

5.1.1 Implications of Detection Probabilities . . . . . . . . . . . . . . 38

5.2 Number of Samples Required to Detect Contamination . . . . . . . . . . . 42

5.3 Number of Samples Required to Characterize Contamination . . . . . . . 46

5.3.1 Hypothetical Situation 1: Small spots of contamination . . . . . . 46

5.3.2 Hypothetical Situation 2: Fairly large region heavily contaminated 47

5.4 Post-Decontamination Sampling _ . . . . . . . . . . . . . . 48

5.4 .1 Experience . . . . . . . . . . . . . . . . 48

5.5 Recommendations . . . . . . . . . . . . . . . 51 
6 Decontamination $\quad 53$

6.1 Decontamination Technologies . . . . . . . . . . . . . . . 53

6.1.1 Surface-Applied Technologies ..................... 53

6.1.2 Gas- and Vapor-Phase Technologies . . . . . . . . . . . . . 54

6.2 Experience . . . . . . . . . . . . . . . . . 55

6.2.1 Hart Building . . . . . . . . . . . . . . . 56

6.2.2 Brentwood Postal Facility . . . . . . . . . . . . . 57

6.2 .3 Sterling Mail Facility . . . . . . . . . . . . . . . . . . . . 57

6.2.4 Other anthrax decontamination experience .......... 58

6.3 Selecting a Decontamination Method . . . . . . . . . . . . 59

6.3 .1 Chlorine dioxide . . . . . . . . . . . . . . 6 60

6.3 .2 Hydrogen Peroxide Vapor . . . . . . . . . . . . . . . . . . . . . 61

6.3.3 Recommendations . . . . . . . . . . . . . . . . . 61

6.4 Acknowledgements . . . . . . . . . . . . . . . . . . . . . 61 


\section{Chapter 1}

\section{Executive Summary}

The goal of this project was to answer the following questions concerning response to a future anthrax release (or suspected release) in a building:

1. Based on past experience, what rules of thumb can be determined concerning: (a) the amount of sampling that may be needed to determine the extent of contamination within a given building; (b) what portions of a building should be sampled; (c) the cost per square foot to decontaminate a given type of building using a given method; (d) the time required to prepare for, and perform, decontamination; (e) the effectiveness of a given decontamination method in a given type of building?

2. Based on past experience, what resources will be spent on evaluating the extent of contamination, performing decontamination, and assessing the effectiveness of the decontamination in a building of a given type and size?

3. What are the trade-offs between cost, time, and effectiveness for the various sampling plans, sampling methods, and decontamination methods that have been used in the past?

\subsection{How much sampling is needed to decide if a building is safe?}

After suspected anthrax contamination, sampling must be performed to determine whether a building needs to be decontaminated. If a building is found to be unacceptably contaminated - contaminated to a level that risks infection of present or future occupants - then more sampling will be needed to characterize the contamination: to determine the spatial extent and the degree of contamination, in order to determine what portions of the building may need special treatment (such as surface-applied decontamination rather than fumigation) and to select decontamination methods.

Even a fairly large number of negative samples may not indicate that a building is safe, because even very low levels of contamination can be dangerous and because contamination may be very localized. To determine the number of samples necessary in order to confirm that a building's level of contamination is safe to within a certain level of risk, we must do the following: 
1. Establish an acceptable level of risk.

2. Convert the risk to a time-average airborne spore concentration, via an assumed dose-response curve.

3. Convert the airborne spore concentration to a spatial-average surface concentration, using an assumed resuspension rate.

4. Convert the surface concentration to a probability that any single sample is positive for anthrax, using a sampling effectiveness curve.

5. Find the total number of samples needed, for the single-sample probability determined in the previous step, such that if all the samples are negative then the building is "safe" with a specified certainty (such as 0.999 probability). A single positive sample is assumed to be enough to assert that the building is contaminated, although in practice confirmatory sampling should of course be performed.

Unfortunately, both the relationships between surface concentration and exposure, and between exposure and risk, are uncertain by a factor of at least 100. The relationship between exposure and risk is particularly uncertain.

\subsubsection{Sampling Nomogram}

We have constructed a graphical method that shows the relationship between risk and the number of samples necessary to ensure that the risk is acceptable. Decision-makers must first determine what level of risk they consider acceptable. (Zero risk is not an option because, as discussed in the body of the report, it cannot be attained). Once an acceptable level of risk is decided upon, Figure 1.1 allows the calculation of the necessary number of samples: it connects the risk from a 1-hour exposure to the number of samples that must be taken in order to confirm that a building is safe, with a specified level of certainty. Begin in the upper left portion of the figure by selecting an acceptable risk of infection for a person who will occupy the building for one hour. Read downward to a dose-response curve, then over to the upper right plot to a "resuspension" curve. Continue downward into the plot below to a sampling effectiveness curve. Finally, read over to the plot at the bottom right, to a curve corresponding to probability of 0.99 or 0.999 of obtaining at least one positive sample. Read off the resulting $\mathrm{x}$-value to determine the number of samples needed. An example is shown for an acceptable risk of $10^{-7}$ per hour of exposure, low risk for a given dose, a high resuspension rate, and a typical real-world sampling sensitivity. In this case, to have a 0.999 chance of detecting contamination at the "acceptable" risk level would require about 80 samples.

Attempting to be very "conservative" (i.e. highly protective) in all of the sub-decisions - the choice of an acceptable risk, the dose-response relationship, the resuspension rate, the assumed sampling effectiveness, and the level of certainty that a building is "safe enough" — will lead to infeasible or even ludicrous decisions. For example, choosing $10^{-8}$ as an acceptable risk of infection for one person-hour of building occupancy, and assuming high risk per dose and a high resuspension rate, will lead to the conclusion that even 0.1 spores per square meter is an unacceptable level of contamination. Standard 
sampling methods cannot detect that low level of contamination. The probability of detecting such a low level of contamination cannot be determined accurately (see the lower right panel of Figure 3.3) since it requires extrapolation to surface concentrations far lower than those investigated experimentally, but the probability of getting a positive sample from any single measurement at such low concentrations is probably less than $10^{-3}$. Thousands of measurements could be made in a building contaminated at this level, without necessarily finding a positive result.

Figure 1.1 assumes that the building is uniformly contaminated (and that the resuspension rate is also uniform), so that the risk is equal everywhere in the building. If the level of contamination were really uniform throughout the building, it would not matter where the samples are taken. If, more realistically, the level of contamination is high in some regions and low in others, the average risk can be the same as in the uniform case, but the number of samples required can be smaller. For example, suppose $90 \%$ the building is completely uncontaminated, and $10 \%$ is highly contaminated. A grid sampling scheme or other system that causes samples to be taken in the highly contaminated region will be certain to detect the contamination, so fewer than 10 samples might suffice. In contrast, if the contamination were spread evenly throughout the building, so that the contamination is near the detection limit everywhere, then even a large number of samples might not result in detection.

Most scenarios that contaminate a building would yield spatially non-uniform contamination, there is no advantage to taking multiple measurements in the same location, and there is a large potential advantage to sampling at spatially dispersed sites, so a grid sampling method or other approach to taking spatially distributed samples should certainly be used. 


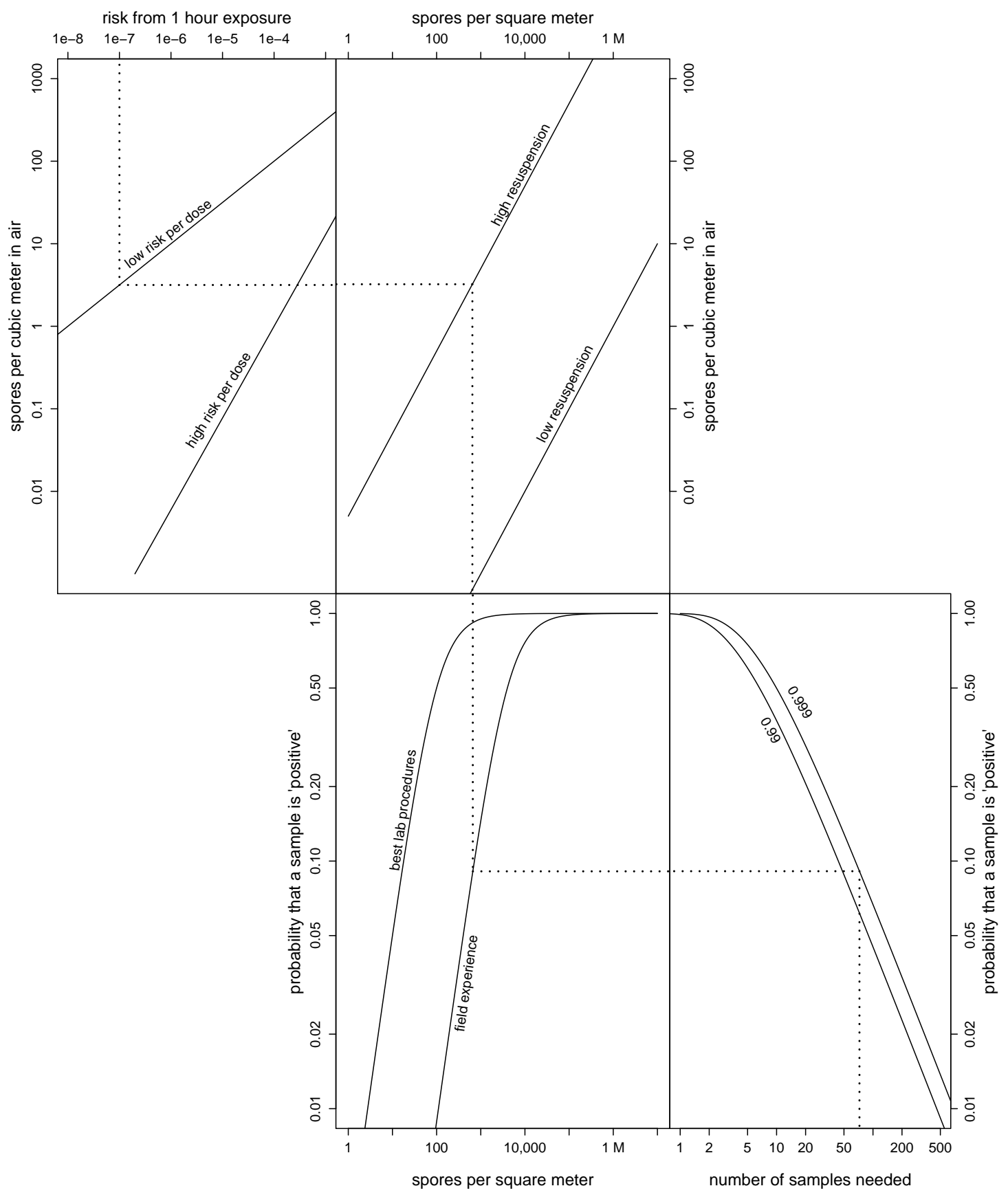

Figure 1.1: Determine the number of samples needed to ensure occupant safety: begin in the upper left portion of the figure by selecting an acceptable risk of infection for a person who will occupy the building for one hour. Read downward to a dose-response curve, then over to the upper right plot to a "resuspension" curve. Continue downward into the plot below to a sampling effectiveness curve. Finally, read over to the plot at the bottom right, to a curve corresponding to probability of 0.99 or 0.999 of obtaining at least one positive sample. Read off the resulting x-value to determine the number of samples needed (all of them negative) to be sure the desired safety level is attained. 


\subsection{How many characterization samples should be taken?}

The goal of characterization is to affect decisions. The quantity and spatial extent of contamination will determine the type of decontamination that will be performed (local surface decontamination versus fumigation) and the physical size of the decontamination task (a single office, a wing of a building, an entire building). A key to effective resource allocation is to make sure that characterization focuses on those goals. For example, once an area has been determined to be heavily contaminated, there is no point to collecting more samples there.

The following are some rules of thumb to help select the number of characterization samples:

1. Begin by estimating the spatial variability of the contamination you are characterizing. If the contamination was brought in by air flow from a distant release, either directly or via the HVAC system, then the spatial distribution will be fairly uniform and there is no advantage to taking samples within a few meters of each other. If, on the other hand, the contamination was carried to specific locations (e.g. a contaminated object was carried through the building and put down at various spots) then a much higher sampling density might be required.

2. Once you have determined that an area (a desk, an office, a suite) is heavily contaminated, there is no need to characterize the contamination in detail: you have already confirmed that the area needs a high degree of decontamination. You are probably better off taking fewer characterization samples, and more samples to confirm decontamination effectiveness. See guidelines for post-decontamination confirmation.

3. Most important, remember that you will be doing post-decontamination confirmation. It is much more important to know that an area is safe after decontamination than to know exactly how unsafe it was before decontamination.

None of these rules of thumb were followed in 2001. There was no systematic attempt to characterize the spatial variability of the contamination; contaminated areas were repeatedly resampled; and because of repeated resampling of areas already known to be contaminated, the ratio of characterization effort to post-decontamination confirmation effort was much higher than it should have been.

\subsection{What decontamination method should be used?}

Considerations for selecting a decontamination method include the following:

1. The probability that decontamination will be successful.

2. The cost of equipment and materials.

3. The ability to monitor the success of the decontamination.

4. The cost of transport and installation of systems (such as pipes and reactors), if necessary. 
5. The personnel effort required, including training time.

6. The resulting damage to building or contents, through corrosion, bleaching, etc.

7. The safety of residue, if any.

This list of considerations will not change for future events, but the method or methods that best meet the criteria will depend on circumstances and will change as technology changes.

For whole-building fumigation, chlorine dioxide gas and hydrogen peroxide vapor remain the most attractive options at the end of 2007. Both can be handled safely, are known to be effective against anthrax, and are readily available. Chlorine dioxide apparently has an edge in effectiveness, because hydrogen peroxide breaks down in the presence of galvanized steel (as in ventilation ducts). However, hydrogen peroxide has an edge in convenience, because it is easier to generate and to work with and because it does not create a substantial volume of liquid waste.

For surface treatment, quite a few products are available, ranging from dilute common bleach to specially formulated foams. The foams may have an advantage in certain circumstances, such as vertical surfaces for which liquids do not have enough contact time, but liquid chlorine dioxide is inexpensive, effective, and easy to use, so it may be the most attractive option. See the EPA report "Compilation of Available Data on Building Decontamination Alternatives" (SAIC, 2005) for information about specific agents. A list and brief descriptions are also provided later in this report.

\subsection{Post-decontamination sampling}

If fumigation is to be performed, then:

1. At least a few known "hot spots" should not be decontaminated with surface methods before fumigation. These hot spots can then be used to help evaluate fumigation effectiveness: if fumigation doesn't completely inactivate the known hot spots, it cannot be trusted to have inactivated unknown areas. It might still be worthwhile to perform additional surface decontamination of known hot spots, even after fumigation that was apparently successful, just to be sure, since surface decontamination of small area is not very expensive or time-consuming.

2. Post-fumigation sampling should be performed at the locations of known "hot spots", and also at some other locations. There is no point in sampling locations where the pre-fumigation surface concentration is known to be low, so concentrate samples in areas that were previously unsampled, undersampled, or found to have relatively high surface concentrations. The exception is areas where the effectiveness of fumigation is not trusted, because of distance from the injection point, proximity to a leak from a non-fumigated area, or a challenging surface for fumigation. Such areas should be sampled even if they did not have particularly high levels of pre-fumigation contamination. 
3. Biological indicators (also known as coupons or spore strips) with known levels of contamination (of a simulant for anthrax) should be used to confirm fumigation effectiveness. Fumigation effectiveness can be affected by temperature, humidity, type of surface, and other environmental factors, but most of these do not vary sharply from location to location. There is no need to place the strips closer together than the distance over which the fumigation effectiveness can change substantially; in most cases this will be at least several meters spacing, and perhaps more. To be "conservative" (i.e. to err on the side of underestimating the fumigation effectiveness), coupons should be prepared so that the kill rate for the coupons is slightly lower than the kill rate for spores deposited in the building; this can be achieved by using a simulant that is hardier (i.e. less susceptible to the fumigant) than anthrax, or perhaps by using a type of coupon material that does not allow the fumigant to be fully effective. Development of coupons or simulants that can predictably fill these roles would be very helpful for future decontamination efforts.

\subsection{What are rules of thumb for cost and effort?}

We had hoped to learn some useful quantitative information about costs, but we were not able to get cost break-downs of the various decontamination projects so as to enable an "apples-to-apples" comparison. For example, the standout in terms of costs seems to be the AMI building: a cost "much less than $\$ 5$ million" is reported for decontaminating a 70,000 square foot office building that had one entire floor that was heavily contaminated, and two other floors with much lower levels of contamination but with spatially dispersed positive samples. This contrasts with, for example, the Hart Senate Office Building, in which most of the $\$ 20$ million decontamination cost went to decontaminating a single 3000 -square-foot office suite. This contrast is misleading, though, because the $\$ 5$ million cost for the AMI building apparently includes only the cost of performing and confirming decontamination of the building per se, whereas the cost quoted for the Hart Building includes many more elements: characterization sampling; disposing of non-critical items; and identifying, handling, preserving, and decontaminating critical items. The cost per square foot of decontaminating the Daschle suite was also increased by the need to build barriers to prevent the fumigant from entering other offices. Without a breakdown of personnel time into different tasks, we cannot tell how much time and money was spent installing and operating the fumigation equipment, and how much was spent on the other decontamination tasks.

All of the decontamination efforts following the 2001 anthrax releases shared many procedures, no matter what decontamination methods they used:

1. Segregate building contents into several types, such as (a) critical for decontamination, (b) important, so decontaminate if possible, and (c) unimportant, so discard.

2. For contaminated areas where fumigation will not be performed, perform local decontamination using surface-applied decontamination agent.

3. For areas where fumigation will be performed, remove all furniture and all items. In most cases, carpet was also removed. In some cases this material was destroyed, 
in others it was treated and returned.

4. For areas where fumigation will be performed, create barriers to prevent fumigant from exfiltrating outside the intended area.

5. For areas where fumigation will be performed, place "biological indicators" (spore strips or coupons) that can be used to determine whether decontamination was successful.

6. Perform decontamination.

7. Perform confirmatory sampling.

8. Repeat decontamination if necessary, and re-sample.

EPA (2002) reports several "lessons learned" that might decrease costs in future responses. For example, they report that identifying, handling, and preserving items identified as "critical" — to be treated at any cost — was very time-consuming, with many items being handled multiple times. Initially, many items were identified as critical that clearly were not. Reducing both the number of critical items and the amount of time spent handling each item were identified as possible major improvements. They suggest performing a cost-benefit analysis in future events, to determine which items are economical to remove and decontaminate.

Many decontamination costs will not vary from one decontamination agent to the next, and indeed, the cost of fumigation may not be the dominant cost of the decontamination effort. The procedures listed above must be carried out no matter what decontamination method is selected: identifying and separating "critical" items, destroying and disposing of contaminated non-critical items, and so on. Lost work days, the loss or destruction of important but perhaps non-critical items, disruption of work, etc., may cost more than the decontamination per se. Future decontamination efforts should put high priority on reducing the costs of work stoppages, materials handling, etc., since such efforts are likely to be more effective at reducing overall incident costs than is effort put into reducing the cost of decontamination per se.

\subsection{Conclusions}

The decision of what level of contamination is unacceptable is critical, since it is a major factor controlling many other key decisions, including (1) the amount of sampling that must be performed to determine if a building is contaminated; (2) the extent of decontamination that must be performed; (3) the decontamination method that should be used; (4) the quantity of building contents that, for cost reasons, should be destroyed rather than decontaminated; and (5) the amount and type of post-decontamination sampling that must be performed to confirm that decontamination was successful.

Compared to the 2001 anthrax response, future responses should take more samples to detect contamination (to ensure that buildings are not falsely declared safe, as the Sterling facility was), and should take fewer samples to characterize contamination (because once it is determined that a portion of a building must be decontaminated, there is little point to taking many additional samples). 
Existing decontamination methods are adequate for the task of decontaminating buildings following an anthrax release: chlorine dioxide gas and hydrogen peroxide vapor are effective, and the cost of their use is likely to be a small percentage of the overall sampling-and-decontamination effort. 


\section{Chapter 2}

\section{Introduction}

In October and November of 2001, contaminated letters were sent to several news outlets and to members of the U.S. Congress. Twenty-two people became infected with anthrax - eleven cutaneous, eleven inhalational - and five of them died: three postal workers, a photo editor, and an elderly retired woman. Contaminated letters were known to have been sent through the mail and to have caused cross-contamination of other letters, but the exact number of contaminated letters was not known, nor their destinations, nor the spatial extent of contamination in contaminated buildings. Therefore, in addition to buildings that were known to have received contaminated letters, many other buildings were sampled. Postal facilities were not initially thought to be at risk, but in fact mail processing proved to release substantial quantities of anthrax spores into the air, and seven postal workers became infected.

In the end, twenty-six buildings on Capitol Hill were sampled, as were a very large number of post offices and mail handling facilities. In at least one case - the State Department Mail Sorting Facility in Sterling, Virginia - initial testing did not succeed in detecting contamination, and this rather heavily contaminated building was not determined to be contaminated until the fifth round of testing.

The following buildings (with short versions of their names, which we use later in this report, in parentheses) were identified as contaminated, and were subsequently decontaminated:

1. American Media, Incorporated (AMI), in Florida;

2. Hart Senate Office Building (Hart Building), in which a letter containing anthrax was opened in the office suite of Senator Tom Daschle (Daschle Suite);

3. Russell Senate Office Building (Russell Building), Washington, DC;

4. Dirksen Senate Office Building (Dirksen Building), Washington, DC;

5. Longworth House of Representatives Office Building (Longworth Building), Washington DC;

6. Ford House of Representatives Office Building (Ford Building), Washington, DC;

7. Brentwood mail sorting facility (Brentwood) in Washington, DC; 
8. State Department mail annex in Sterling, VA (Sterling);

9. P Street mail warehouse (P Street), Washington, DC;

10. CBS Building, New York, NY.

11. GE Building, NBC News offices, New York, NY.

The level of contamination found in these buildings ranged from very high (in the Daschle Suite and on some Brentwood mail sorting machines) to very low (in the CBS Building).

This report examines several aspects of the response to the 2001 anthrax attacks, and recent research in anthrax sampling and decontamination, to develop guidance for use in future anthrax incidents. We begin by discussing the decision of what level of anthrax contamination to accept in a building: considering the dose-response relationship for anthrax, and the relationship between surface and airborne contamination, what degree of contamination must a sampling scheme be able to detect? The answers to those questions affect the number of samples and the spatial distribution of samples that must be taken, as we discuss.

We then investigate the spatial distribution of contamination, using observations from the 2001 anthrax releases as well as results from a simple predictive model. We discuss the spatial pattern of contamination in the AMI building in some detail, and also summarize what is known about the distribution in other buildings.

We continue by discussing sampling technologies and effectiveness, including the proportion of false negative results. We analyze the sampling characteristics together with results from the prior chapters so as to generate advice about sampling plans. For example, in the response to the incidents of 2001, a great deal of effort went into "characterization sampling," to generate a detailed quantitative description of contamination in buildings once they had been found to be contaminated. Our analysis finds that future responses should perform less characterization sampling: once a high degree of contamination has been confirmed, there is little point in performing additional sampling, since the results of heavier samplings will not affect decisions such as whether or not to perform large-scale decontamination.

Finally, we discuss briefly the effectiveness, costs, and pros and cons, of various decontamination methods, and recommend specific methods for use in future anthrax responses. 


\section{Chapter 3}

\section{How Clean is Safe?}

The decision of what level of contamination is unacceptable is critical, since it is a major factor controlling many other key decisions, including (1) the amount of sampling that must be performed to determine if a building is contaminated; (2) the extent of decontamination that must be performed; (3) the decontamination method that should be used; (4) the quantity of building contents that, for cost reasons, should be destroyed rather than decontaminated; (5) the amount and type of post-decontamination sampling that must be performed to confirm that decontamination was successful.

The "how clean is safe" decision has at least two separate components. First, what level of risk is acceptable, i.e. what is an acceptable probability that a building occupant will contract anthrax? Second, what level of contamination guarantees that risk is below the acceptable level?

This latter question requires a way of estimating inhalation exposure as a function of the surface concentration of anthrax, as well as a way of estimating the risk of contracting anthrax from a given amount of inhaled anthrax. Unfortunately, both relationships are uncertain by a factor of at least 100, as we discuss later.

Importantly, "zero risk" and "zero contamination" are not reasonable standards to apply, since they cannot be guaranteed at any cost. As we discuss later, there is at least a possibility that a single inhaled spore can cause anthrax, with some extremely low probability. Since it is always possible for any single spore in a building to become airborne and to be inhaled, even a single spore in a building presents a non-zero risk (albeit very small). But there is no sampling technology that can conceivably guarantee the detection of a single spore if it is present. So it is not possible, even in principle, to sample a building sufficiently to guarantee that there is no risk to occupants.

With the sampling techniques and technologies applied in 2001, low spatial densities of anthrax spores (under about 1000 "colony-forming units" per square meter) were very unlikely to be detected by surface sampling. Even buildings that tested negative for anthrax may have had a moderate amount of anthrax contamination. There was no option other than to leave buildings contaminated if they had concentrations at these levels - barring expensive decontamination of buildings that had no positive tests because the contamination could not be detected.

As technologies and techniques improve, previously undetectable concentrations of anthrax will become detectable. At some point, it might reasonably be considered too 
expensive or disruptive to decontaminate an area with a very low, but detectable, level of contamination. The question - and it is a question that drives many decisions concerning sampling and decontamination - is what level of contamination is considered to be acceptable.

A rational decision on the acceptable level of surface contamination should, of course, be based primarily on the risk posed by the contamination. Most of the risk from anthrax is due to the risk of inhaling spores: "inhalational" anthrax, which is contracted by inhaling spores, is much more dangerous than "cutaneous" anthrax, which is contracted through skin contact. The risk to a building occupant from a given density of spore surface loading depends on two relationships: the relationship between surface loading and airborne concentrations, and the relationship between airborne concentrations and the probability of contracting anthrax. As described above, the concentration of spores on surfaces (combined with other factors) determines the airborne concentration, and the airborne concentration (combined with other factors) determines the risk.

More specifically, the airborne concentration of resuspended anthrax depends on the surface concentration, the level of activity, the type of surface, and the ventilation rate. Unfortunately, the relationship between spore surface loading and the resulting airborne concentrations is uncertain by one or two orders of magnitude, even if the floor type and activity level are known.

The risk from a given airborne concentration depends on the characteristics of the exposed population (the proportion of elderly or young, healthy or ill, etc.). Unfortunately the dose-response relationship is even more uncertain than the relationship between surface and airborne concentrations.

\subsection{Dose-Response Relationship for Anthrax}

Anthrax can lead to two types of infection: "cutaneous" anthrax causes skin lesions, while "inhalational" anthrax attacks the lungs. Inhalational anthrax is far more dangerous than cutaneous anthrax, and is responsible for essentially all anthrax deaths. Untreated inhalational anthrax is very likely to be fatal, and in fact for inhalational anthrax, many authorities do not distinguish between $\mathrm{LD}_{50}$ (a degree of exposure that is lethal to half the people exposed) and $\mathrm{ID}_{50}$ (a degree of exposure that infects half the people exposed).

The does-response relationship for anthrax is extremely uncertain, especially at low dose: there are few circumstances in which a large number of people are exposed to known numbers of anthrax spores. Extrapolation from animals is not very reliable because of differences in immune response and lung morphology between people and animals.

Also unknown is the clinical relevance of clumps of anthrax (known as colony-forming units, or CFU) compared to individual spores: is a clump of 100 spores 100 times more likely to cause infection than a single spore, or only 10 times, or is it equally likely?

Fennelly et al. (2004) summarized results from previous analyses of anthrax healtheffects data, and re-analyzed data from an anthrax outbreak in Manchester, England in 1957 for which some exposure data were available. For a virulent strain of anthrax they estimate $\mathrm{ID}_{25}$, the dose that will infect $25 \%$ of a population, to be $140-690 \mathrm{CFU}$ smaller than $5 \mu \mathrm{m}$ and $620-2200$ total CFU. They estimate ID $_{50}$ to be $1400-6900 \mathrm{CFU}<5 \mu \mathrm{m}$ and $6200-22000$ total CFU. The relative importance of total CFU versus individual 
spores or small groups of spores is not known. Fennelly et al. note that their estimates overlap with those of other studies, including the more or less standard assumption that $\mathrm{LD}_{50}$ is between $2500-55000$ spores. $\mathrm{LD}_{50}$ and $\mathrm{ID}_{50}$ are uncertain by at least a factor of 5 , and the dose-response relationship at lower doses is even more uncertain because it relies on extrapolation by a factor of 100 or 1000 downward in dose.

Glassman (1966), Wilkening (2006), and others (Peters and Hartley, 2002, and Fennelly et al., 2004 for example) suggest that 100 spores or even less might be enough to cause infection in rare cases. Wilkening (2006) discusses five dose-response models. He considers infection models that predict the probability of infection from 100 spores at $10^{-1}$ to $10^{-5}$, and applies these models to an analysis of the Sverdlosk incident. Wilkening suggests that the lower values (i.e. those with less risk at a given exposure) appear to be inconsistent with Sverdlosk data, and that the chance of contracting anthrax from 100 spores may be somewhere in the range of 0.001 to 0.1 .

Two women who contracted inhalational anthrax in 2001 are not known to have been exposed to substantial numbers of spores, and are presumed to have been infected by cross-contamination of mail. There is no good quantitative estimate of their exposure, but it may have been less than 100 spores and perhaps less than 10. One of the women was over 90 years old and the other over 60, so age may have been a factor: there are many reasons the elderly would be expected to be more susceptible (see Fennelly et al. 2004, for example).

Although, as discussed above, there is evidence that even 100 spores or so can cause anthrax at least in particularly susceptible people, data strongly suggest that the risk of contracting anthrax from a very small number of spores - less than 10 or so - is extremely small, probably less than $10^{-4}$ and possibly $10^{-5}$ or even lower:

1. Inglesby et al. (2002) note that "At Sverdlosk, no new cases of inhalational anthrax developed beyond 43 days after the presumed date of release ... despite only limited decontamination and vaccination of [less than $5 \%$ of the city's population.]" Decontamination at Sverdlosk (now known as Ekaterinberg) included decontaminating a ceramics factory with chloramines; spraying streets, pavement, and trees with unspecified disinfectant, scrubbing roofs and walls of some buildings with caustic solutions; removing and burying topsoil in contaminated areas; and resurfacing contaminated dirt roads with asphalt (Sharp and Roberts, 2006).

2. Inglesby et al. (2002) note that "during the first half of the twentieth century, a significant number of goat hair mill workers were heavily exposed to aerosolized spores," but "only 13 cases of inhalational anthrax were reported." Besides these occupational exposures, only 5 additional cases were reported in the entire U.S. in the 20th century. The mill workers' family members and others in the community also must have been exposed to low levels of spores, but did not contract anthrax. The mill workers are estimated to have inhaled several hundred anthrax particles per 8-hour shift (Dahlgren et al., 1960). It has been suggested that prolonged exposure to low concentrations of anthrax builds up immunity, and while this may very well be true, there is still a need to explain why few or no workers became infected in their first week on the job, or why there was no anthrax among friends and family who were exposed to spores tracked home on clothes or hair. 
3. Dull et al. (2002) and Teshale et al. (2002) found that anthrax was still present, and aerosolizable, on a mail sorting machine even after it had processed more than 1 million pieces of mail following its becoming contaminated. Some of the resulting contaminated mail must have cross-contaminated other mail, and direct contact with the initial anthrax-containing letter must also have contaminated other mail.

Yet only two people contracted inhalational anthrax who were not known to have been exposed to substantial numbers of spores.

The figures above refer to the numbers of viable spores that cause infection. Spore viability will decrease with time, although the rate of loss of anthrax viability indoors is not known. It is known that anthrax spores are extremely hardy and can survive for decades in the right conditions: "The organism may form a spore after local nutrients are exhausted, and can survive in a non-vegetative spore state for many years. The bacilli have been isolated from infected soil stored for as long as 60 years." (Burrows, 1973, cited in Barth et al., 2003). Sharp and Roberts (2006) document several anthrax outbreaks in cattle in Great Britain that apparently were caused by spores that were dormant for years or decades.

Anthrax viability depends on environmental conditions, and viability will decrease with time, but there is very little quantitative information about this, and many models are consistent with what is currently known. Stuart and Wilkening (2005) summarized research on this issue and find that, although a reasonable estimate can be made for the decay rate of anthrax viability over the first hour or two after release into the environment, there is enormous uncertainty over longer times.

We do know that, in spite of the longevity of anthrax spores and the apparently nonnegligible risk of infection from even a small number of them, there have been very few, or perhaps no, cases of inhalation anthrax caused by spores resuspended from building surfaces. All of the anthrax victims in the 2001 attacks were either directly exposed to spores immediately following an airborne release from opened or disturbed mail, or are believed to have been exposed via contaminated mail. And as noted above, no one contracted anthrax in the months following the Sverdlosk release, in spite of the fact that decontamination efforts there could not have inactivated all of the spores.

\subsection{Relationship between Surface Concentration and Dose}

Anthrax spores deposited on surfaces do not present a direct threat of inhalation anthrax: they must first be resuspended into the air so that they can be inhaled. The relationship between surface and airborne concentrations depends on the type of surface and on the activity that causes resuspension.

Several experiments have quantified the relationship between airborne and surface concentrations of biological agents. A study at Dugway concluded that with ground contaminated with 20 million Bacillus subtilis spores per square meter, a soldier exercising actively for a 3-hour period would inhale between 1000 and 15000 spores. (Resnick et al., 1990, cited in Inglesby et al. 2007). This suggests that the number of spores per cubic meter in the breathing zone can be obtained by multiplying the surface concentration (in spores per square meter) by about $10^{-5} / \mathrm{m}-10^{-3} / \mathrm{m}$. 
Buttner et al. (2002) performed lab experiments with Penicillium chrysogenum spores resuspended by walking, and found large variation in resuspension rates between different floor surfaces: $4 \times 10^{-4}-3 \times 10^{-3} h^{-1}$ for vinyl tile, $5 \times 10^{-5}-3 \times 10^{-3} h^{-1}$ for commercial carpet, and $0.05-0.2 h^{-1}$ for residential carpet.

We would rather work with airborne concentrations than with numbers of particles, and Buttner's resuspension rates do not directly translate into airborne concentrations because (1) the airborne concentration also depends on the removal rate of particles from the air, and (2) the number of airborne particles must be divided by the volume of air. Let $N_{d}$ be the number (not concentration) of deposited particles, $N_{a}$ the number of airborne particles, $r$ the resuspension rate as determined by Buttner et al. (2002), and $f$ the removal rate. Then

$$
N_{a}=\frac{r N_{d}}{f}
$$

which, for a room with floor area A and volume $\mathrm{V}$, yields an airborne concentration

$$
C_{a}=\frac{r A}{f V} C_{d}
$$

where $C_{d}$ is the number of particles per square meter on the floor. For small particles (diameter $<5 \mu \mathrm{m}$ ), the deposition rate is very low and ventilation is the dominant removal mode. Ventilation rates in most rooms are in the range of $f=1-10 h^{-1}$. Buildings typically have a volume to surface ratio of about $V / A=3 \mathrm{~m}$. Thus $A / f V \approx 0.1 / \mathrm{m}$, which can be multiplied by the results of Buttner et al. (2002) to predict approximately the relationship between surface and airborne concentrations: the airborne concentration of spores (particles per cubic meter) can be determined by multiplying the surface concentration (particles per square meter) by about $10^{-3} / \mathrm{m}$ to $10^{-6} / \mathrm{m}$, depending on the type of surface and the level of activity. The higher values apply to carpet, and the lower to vinyl tile. This range of factors is similar to the range determined by Resnick et al. for outdoor activity, as described above. This correspondence is not necessarily expected a priori, since the surface characteristics and the air flows differ greatly between indoors and outdoors.

Weis et al. (2007) measured surface particle concentrations (Table 3.2) and re-suspended concentrations during simulated office activities in the Daschle suite of the Hart building (Table 3.2). The measured airborne concentrations in the entrance area during the active period were roughly 100 particles per cubic meter in the breathing zone, and 1000 at floor level. Activities that generated these levels are not presented in detail; Weis et al. say "active office conditions were simulated to reflect routine behaviors in a busy office environment (i.e., paper handling, active foot traffic, simulated mail sorting, moving trash containers, patting chairs)." Surface loading in the entrance area was not directly measured, but outside the Mail Area there was only moderate measured variability in surface concentration, with concentrations (estimated by vacuuming the carpet) ranging from about 1000 to $4500 \mathrm{CFU}$ per $100 \mathrm{~m}^{2}$, or $10^{5}$ to $4 \times 10^{5} \mathrm{CFU}$ per $\mathrm{m}^{2}$. So, empirically, multiplying the spatial concentration (in particles per $\mathrm{m}^{2}$ ) by about $3 \times 10^{-3} / \mathrm{m}$ yields the breathing height concentration (in particles per $\mathrm{m}^{3}$ ). Multiplying the surface concentration by about $2 \times 10^{-2} / \mathrm{m}$ yields the airborne concentration at floor level. All of the carpet concentrations are based on the number of spores recovered; the actual 
Table 3.1: Table from Weis et al. (2007): Surface anthrax concentrations (CFU per $100 \mathrm{~cm}^{2}$ ) measured in various locations in the Daschle suite. TNC $=$ Too Numerous to Count.

\begin{tabular}{|l||rrrr|}
\hline Location & Carpet & $\begin{array}{r}\text { Fabric } \\
\text { office } \\
\text { dividers }\end{array}$ & $\begin{array}{r}\text { Smooth } \\
\text { horizontal } \\
\text { surfaces }\end{array}$ & $\begin{array}{r}\text { Tops } \\
\text { of file } \\
\text { cabinets }\end{array}$ \\
\hline Mail Area & TNC & NA & NA & NA \\
Workstation B & 1500 & 200 & 2000 & 0 \\
Workstation C & 4000 & 200 & 6800 & NA \\
Workstation D & 1100 & 100 & 100 & 100 \\
Workstation E & 4600 & 0 & 2500 & 0 \\
\hline
\end{tabular}

surface loading may have been several times higher than these values. If we assume the vacuum method recovered about $1 / 3$ of the spores on the surface, then multiplying the actual spatial concentration by about $10^{-3} / \mathrm{m}$ would yield the approximate breathing height concentration. This is consistent with the upper end of the resuspension estimates of Buttner et al. (2002) and Resnick et al. (1990), discussed above. All of the investigations agree that multiplying the surface density of spores (particles per square meter) by $10^{-6} / \mathrm{m}$ to $10^{-3} / \mathrm{m}$ yields the airborne concentration (particles per cubic meter) if there is a moderate level of disturbance of the surface.

\subsection{Setting a Safety Standard}

Figure 3.3 shows the relationship between risk, airborne spores, and surface spores, for one hour of exposure. Dose-response curves correspond approximately to those of Wilkening (2006). For the relationship between airborne and surface concentrations, we chose a high-resuspension case to roughly match the high-resuspension conditions measured by Buttner et al. (2002), and to be about ten times higher than the values implied by the work of Weis et al. (2007). Even higher resuspension rates could plausibly be generated by some activities, such as vacuuming, although these activities would likely be short-lived and local.

We have no data on the concentration that would be induced by a high level of disturbance, such as vacuuming a carpet or sweeping a vinyl floor, but it seems likely that the airborne particle concentrations would be substantially higher than in the case of moderate disturbance. Of course, vacuuming and sweeping are normally short-duration disturbances in any single location, so occupants of a space might experience the resulting elevated particle concentrations for only a few minutes. However, the people performing the sweeping or vacuuming might be exposed to elevated concentrations for much of their workday, so special consideration should be given to their safety even in a building that is safe for most occupants.

Figure 3.3 assumes one hour of exposure to airborne spores. To attain the same level of risk in the case of a longer exposure time, such as ten hours, requires decreasing 
Table 3.2: Table adapted from Weis et al. (2007): Airborne anthrax concentrations (Number of Colony Forming Units trapped by a $4 \mathrm{~L} / \mathrm{min}$ air sampler), measured in the Daschle Suite of the Hart Building. The original table shows both direct measurement results and results adjusted for sampling bias; only the adjusted numbers are shown below, rounded to appropriate significant figures.

\begin{tabular}{|c|c|c|c|c|c|c|}
\hline \multirow{5}{*}{$\begin{array}{c}\text { Nominal } \\
\text { particle size }(\mu \mathrm{m})\end{array}$} & \multicolumn{6}{|c|}{ Number of CFUs } \\
\hline & \multicolumn{3}{|c|}{ Mail Handling Area } & \multicolumn{3}{|c|}{ Entrance Area } \\
\hline & \multirow{3}{*}{$\begin{array}{r}\begin{array}{r}\text { Semi- } \\
\text { quiescent }\end{array} \\
\text { Floor } \\
\text { Level }\end{array}$} & \multicolumn{2}{|c|}{ Active Conditions } & \multirow{3}{*}{$\begin{array}{r}\begin{array}{r}\text { Semi- } \\
\text { quiescent }\end{array} \\
\text { Floor } \\
\text { Level }\end{array}$} & \multicolumn{2}{|c|}{ Active Conditions } \\
\hline & & Floor & Breathing & & Floor & Breathing \\
\hline & & Level & Zone & & Level & Zone \\
\hline$>7.1$ & 1 & 71 & 3 & 0 & 13 & 0 \\
\hline $4.71-7.1$ & 0 & 19 & 13 & 2 & 9 & 0 \\
\hline $3.01-4.7$ & 2 & 61 & 13 & 5 & 12 & 1 \\
\hline $2.01-3.0$ & 3 & 110 & 20 & 9 & 31 & 3 \\
\hline $1.01-2.0$ & 31 & $>2600$ & 90 & 40 & 115 & 24 \\
\hline $0.65-1.0$ & 11 & 115 & 61 & 13 & 24 & 2 \\
\hline Total CFUs & 50 & $>3000$ & 200 & 70 & 200 & 30 \\
\hline $\begin{array}{c}\text { Estimated air } \\
\text { concentration } \\
\left(\mathrm{CFU} / \mathrm{m}^{3}\right)\end{array}$ & 170 & $>11000$ & 710 & 250 & 720 & 110 \\
\hline
\end{tabular}

the airborne concentration by a factor equal to the number of hours. For example, the high-risk-per-dose curve implies that a risk of $10^{-7}$ occurs at an airborne concentration of about 3 spores per cubic meter, for one hour; about the same risk would occur at an airborne concentration of 0.3 spores per cubic meter for ten hours.

Extrapolating to a much longer period, such as a month or a year, has several substantial additional complications: (1) the number of viable spores per square meter, and the concentration of viable airborne spores, will not remain constant, since (a) some spores will lose their viability and (b) spores will be removed from the building through the HVAC system, tracking on shoes or clothes, vacuuming, etc.; and (2) prolonged exposure to very low levels of spores may promote immunity and thus alter the dose-response with time (towards lower risk per dose). Removal mechanisms and loss of viability will lead to risk from prolonged exposure that is lower than predicted by multiplying a short-duration risk by the number of hours of exposure. 


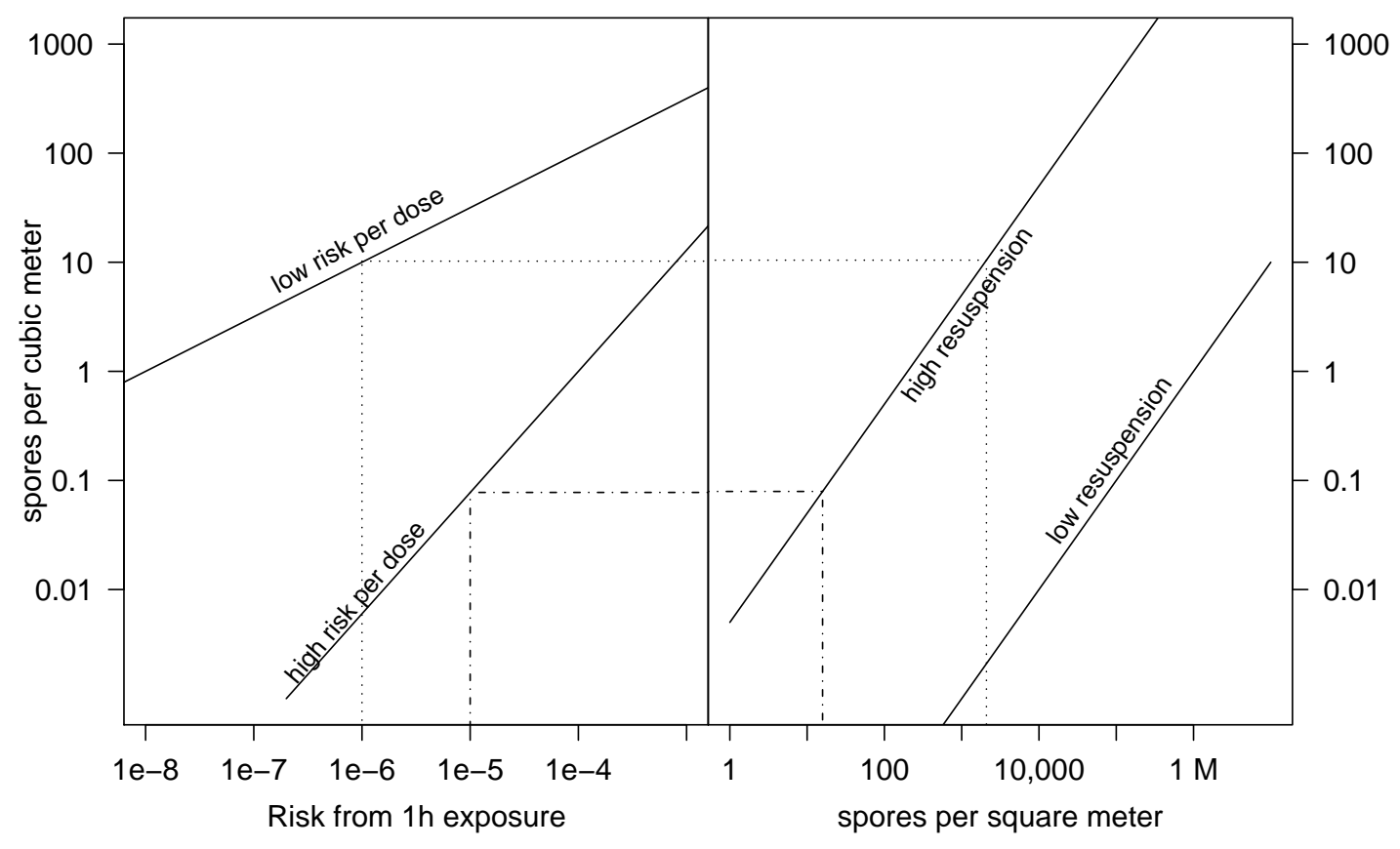

Figure 3.1: Plot for determining the surface concentration that corresponds to a given level of risk of infection, for someone who stays in the building for one hour. Begin by choosing a risk on the $\mathrm{x}$-axis of the left side of the figure. Go up from that risk value until reaching the dose-response line of your choice. (Two dose-response lines are shown; almost any monotonically increasing curve between these lines is also consistent with data on anthrax infection). The corresponding y-value is the number of spores per cubic meter that give rise to the chosen risk level. Now move along that y-value onto the rightmost plot, until reaching the resuspension line of your choice. The corresponding $\mathrm{x}$-value is the surface concentration that gives rise to your chosen risk value. The procedure may be performed in reverse to determine the risk caused by a given surface concentration. Examples are shown for two cases: (1) risk of $10^{-6}$, assuming the low-risk-per-dose relationship and high resuspension rate, and (2) risk of $10^{-5}$, assuming high risk-perdose and high resuspension rate. Depending on the dose-response relationship, even a low surface density of spores can cause an unacceptably high risk. 


\section{Chapter 4}

\section{Spatial Distribution of Contamination}

Several phenomena control the spatial variability of concentrations of anthrax deposited in a building. These include:

1. Anthrax may be introduced to a building in different ways: objects with surface contamination may be brought into the building (letters, packages, etc.) and distributed throughout the building; anthrax may be introduced to the air at a single location through a dispersal device (a powder-filled letter or package may be opened, or a dispersal device may be triggered); anthrax may be introduced to the air at multiple locations; anthrax may be introduced to the ventilation system through the building's air intakes; anthrax may enter the building from an outdoor plume. Each of these will produce a different spatial pattern of contamination.

2. The ventilation system will spread airborne contamination around the building. The resulting spatial distribution will depend on the amount of air exchange between various portions of the building, the amount of air that is recirculated into the building rather than being exhausted to the outdoors, and the efficiency of filters in the ventilation system at removing particles the size of the anthrax spores or clusters.

3. After introduction to the building, anthrax can spread as people carry contaminated objects through the building, and as deposited anthrax is re-aerosolized through disturbance of surfaces (walking, shuffling papers, vacuuming, etc.) and then carried by air flows.

As long as a substantial fraction of the anthrax is airborne, air flows are the dominant transport mechanism. The surface concentration that results from the spread of airborne particles will change gradually as a function of location within each room, but with a high concentration near the release area since the larger particles will rapidly settle there. Smaller particles will disperse more widely, at least in rooms near the release and (depending on the type of filter in the HVAC system, and whether the system recirculates indoor air) possibly throughout the entire HVAC zone that serves the release 
area. The locations of walls, and whether doors are open or closed, have a large effect on air flows and thus particle transport, so the surface concentration can be much higher in one room than in an adjacent room. Sajo et al. (2002) and Griffith et al. (2006) (see Figure 4.5, below) give examples of these phenomena. For example, Sajo et al. performed aerosol release experiments in a two-story, 21-room office building served by a single HVAC sytem, using particles with a geometric mean diameter of $1.1 \mu \mathrm{m}$ and a geometric standard deviation of 2 . They found that the density of deposited mass varied by only about a factor of 20 from one sampling location to another through most of the building, but that concentrations an additional factor of 10 higher were found close to the release point.

After a few minutes to a few hours (depending on particle size and ventilation rate), most of the anthrax that remains in the building has deposited on surfaces. Once most of the particles have deposited, tracking (on shoes and clothes), and transport of contaminated items, become significant factors in moving anthrax around the building. Whether air flow continues to play a significant role depends on the resuspension rate: at relatively high resuspension rates (e.g. if $1 \%$ of material is resuspended each hour) air flows will continue to play a significant role, but at lower rates (e.g. $0.001 \%$ resuspended each hour) air flows are probably no longer important because the other transport mechanisms will likely dominate.

If anthrax release is detected quickly, airflow is the most important transport mechanism because people quickly leave the area, so tracking and re-suspension activities stop. This was the case in the Daschle suite of the Hart building in 2001. But for most anthrax releases that are not immediately detected, all of the transport phenomena listed above will come into play. For example, consider the AMI building, which we discuss in detail below. Anthrax was released by the opening of at least one letter (near the mailroom) and possibly a second (in the index case's office) that contained large numbers of anthrax spores. Anthrax spilled directly onto objects and people at the release location(s), and clouds of anthrax particles were released to the air. Some anthrax was spread throughout portions of the building via the HVAC system and via direct air flows between rooms and corridors. Over succeeding days, anthrax spread throughout the entire building as it was tracked on people's shoes and clothes, and as cross-contaminated objects, including mail, were carried through the building. Sweeping, vacuuming, and walking undoubtedly re-suspended some of the spores, which were then carried by the air. The resulting spatial distribution of anthrax included anthrax spread through the ventilation system (probably fairly uniformly, within any single ventilation zone); anthrax spread through direct airflow between the release location(s) and other rooms (and probably much more concentrated near the release locations than far away); and contaminated objects, such as mail, left in some locations (and probably representing pockets of high concentration amidst a lower background).

The situation was qualitatively similar (though quantitatively different) in the several contaminated mail sorting centers. In the Brentwood facility, for example, a cloud of airborne particles was created at Mail Sorter 17, and measurements showed that these airborne particles contaminated surfaces at least $60 \mathrm{~m}$ away. Particles also contaminated nearby mail, and the machine that sorted mail, so that subsequent mail that passed through the machine also picked up some contamination. Contaminated workers also 
tracked contamination through the building.

In this chapter we discuss what is known about the spatial distribution of anthrax in several buildings that were contaminated in the 2001 anthrax attacks, as well as the spatial distribution of particles in an experiment in a building. We also discuss the results of some simple models that quantify, approximately, the difference in spatial concentrations between various areas of a building that is contaminated by a single release at a specific location. The goal is to gain some understanding of the degree of spatial non-uniformity that can be expected.

It would be unwise to rely too heavily on past experience or on modeling. All of the 2001 releases, the experiment, and the modeling, are cases in which anthrax was aerosolized - small particles were ejected into the air — at a single location inside the building and at a single time (although some have speculated that there may have been two releases at the AMI building). Other release scenarios would give rise to different spatial patterns. Anthrax introduced into a building's air intake would probably spread much more uniformly through a building, for example, compared to the 2001 events, which led to very high spatial densities of contamination near the locations where contaminated mail was opened.

\subsection{Hart Building}

Following the opening of an anthrax-containing letter in Senator Daschle's suite in the Hart Office Building, the building was evacuated and sampling was performed. Results from initial sampling indicated measurable anthrax contamination in 17 suites. "The routes of people who potentially had been exposed to the spores were followed up to the ninth floor, then back down to the fifth floor. The stairs and a nearby ladies room were sampled and showed positive results." (EPA 2002, p. 54). The "mail trail" taken by the contaminated letter was also re-traced and sampled. Sampling was also performed in the HVAC system, where "sampling revealed that all HVAC systems were clean, except the Daschle stack, and that secondary contamination of the HVAC system had not occurred." (EPA 2002, p. 54)

The Daschle suite in the Hart building occupies portions of the 5th and 6th floor, with an internal stairwell. Thirteen people who were present near where the letter was opened, on the 6 th floor, tested "very positive" with nasal swabs, 14 hours later. An additional 7 of the 23 who were in Daschle's 5 th floor office also tested positive. Furthermore, 2 out of 18 in Senator Feingold's office (adjacent but not connected) tested positive. Later, 5 out of 9 first responders tested positive, and a security guard posted outside the office when the letter was opened tested positive (Vogt et al. 2002.) The people listed in this paragraph total 28 positives, which is all of the positive tests out of about 400 people on those floors of the building and hundreds of other visitors to the building. A total of about 5000 nasal swabs were collected and tested. 


\subsection{Other Capitol Hill locations}

Longworth Building: Approximately 1450 samples (including initial, characterization, and verification). Initial assessment found anthrax in and around three suites. HVAC systems were sampled and all were negative (EPA 2002, p. 30).

Ford Building: 170 samples in initial assessment. Eight samples collected in four rooms tested positive. Characterization collected 115 additional samples; all tested negative (EPA 2002). After decontamination, "Air verification sampling was conducted ... to closely simulate conditions that would be found ... once the rooms were re-occupied. The air sampling ... included high-volume dry filter units (DFU) and five low-volume gelatin samples. One of the ...gelatin samples returned a positive, indicating that anthrax was re-aerosolized ..." (EPA 2002, p. 33).

Dirksen Building: 130 initial samples; 6 tested positive for anthrax (EPA 2002, p. $35)$.

P Street Warehouse: 42 initial samples; "three areas" tested positive for anthrax (Senate Furniture area, House mail storage area, and four X-ray machines in the loading dock area). 360 additional characterization samples were collected (EPA 2002, p. 36)

Russell Building: 38 initial samples, 228 characterization, and 7 verification, in response to an anthrax-contaminated letter (EPA 2002, p. 39).

\subsection{Brentwood}

Sanderson et al. (2002) report co-located sampling from non-porous surfaces in Brentwood. HEPA and wet wipes had the most positives, and agreed with each other well. Let "HEPA+" and "HEPA-" indicate a positive or negative result from a HEPA vacuum sample, and similarly for Wipe+ and Wipe-. From 58 co-located samples, the Brentwood data reported $44 \mathrm{HEPA}+$ and Wipe+; 4 with HEPA+ and Wipe-; 5 with HEPA- and Wipe+; and 5 with HEPA- and Wipe-. If we assume that there are no false positives, then the cases of HEPA+ / Wipe- (and vice versa) tell us that there are some false negatives.

Consider a very simple model in which there is some (unknown) concentration, below which a sample has a high chance of generating a false negative, and above which a sample is very likely to report a positive. Moreover, assume that the probabilities of a negative or positive result are the same for both HEAP and Wipe samples. For example, using standard notation for conditional probability, assume $P$ (positive|low concentration) $=$ 0.5 and $P$ (positive|non-low concentration $)=1$. If there were 18 low concentrations and 40 non-low concentrations out of the 58 measured locations, then this simple model predicts 44.5 locations with HEPA+ and Wipe+, 4.5 with HEPA+ and Wipe-, 4.5 with HEPA- and Wipe+, and 4.5 with HEPA- and Wipe-. This is in excellent (and probably partially coincidental) agreement with the observations of 44, 4, 5, and 5 respectively. In other words, the Brentwood data reported by Sanderson et al. agree very well with a simple model in which the entire facility sampled by HEPA and Wipe samples was contaminated, at least at a low level, even though there were some cases in which both samples were negative. 
Table 4.1: Number of positive samples and total number of samples (from all sampling methods) in selected areas of the AMI Building, from Traeger et al. (2002).

\begin{tabular}{|l|c|c|r|}
\hline & \multicolumn{2}{|c|}{ Positive / total samples } & Total \\
\hline Location & Oct $8-10$ & Oct 25 - Nov 8 & $1 / 1=100 \%$ \\
\hline Basement ventilation filter & $1 / 1$ & & $9 / 10=90 \%$ \\
Mail-sorter office & $5 / 6$ & $4 / 4$ & $13 / 26=50 \%$ \\
Mailroom & $10 / 20$ & $3 / 6$ & $4 / 39=10 \%$ \\
Text library & $1 / 9$ & $3 / 30$ & $2 / 24=8 \%$ \\
Index patient work area & $2 / 21$ & $0 / 3$ & $2 / 3$ \\
\hline
\end{tabular}

At the Brentwood mail facility, a cone-shaped filtering sock (manufactured by Midwest Filtration Co., Fairfield, Ohio) was inserted into the nozzle of a HEPA vacuum cleaner (Vacomegah, manufactured by Atrix International Inc., Burnsville, Minnesota). Wipe samples were also performed. Details of the procedures are in Sanderson et al. (2004). In the first survey (23-28 October 2001), 8/114 $=8 \%$ of the wipe samples were positive for anthrax and $27 / 39=69 \%$ of HEPA samples were positive. Details of the spatial distribution of all of the samples, and the methods used to collect them, are given in Table 5.1.

The Brentwood data show unambiguously that anthrax spores spread large distances through the air: Samples from window portals $5 \mathrm{~m}$ above floor level were contaminated. Window portals above DBCS machine 17 were heavily contaminated. The contamination on portals decreased rapidly beyond $60 \mathrm{~m}$ from DBCS 17 . No spores were detected on portals more than $90 \mathrm{~m}$ from the machine (Sanderson et al., 2004).

\subsection{American Media, Inc. (AMI) Building}

The exact circumstances of the release or releases of anthrax in the AMI building are not known. Traeger et al. (2002) speculate that two envelopes containing anthrax may have been opened in the building on or around September 25. One of the envelopes is believed to have been opened in an office near the mailroom, and one of them in an office on the third floor that contains the work area of the first patient to be diagnosed with anthrax (the "index patient"). A mailroom worker also contracted anthrax. Anthrax was diagnosed in the index patient on October 2, 2001, and was confirmed from fluid culture on October 4. Initial sampling was performed from October 8-10.

Samples were taken using several protocols including various types of wipes, swabs, and vacuum methods. An initial set of samples was taken during October 8-10, 2001, to determine whether the index patient had contracted anthrax in the building and to begin to determine the level of contamination if any. The initial samples confirmed that the building was contaminated, and the building was turned over to the Federal Bureau of Investigation (FBI) for forensic testing. We were not able to obtain data from the FBI tests. After the FBI vacated the building, another intensive round of testing was done, under the guidance of the Centers for Disease Control, and most of our discussion is based on those tests. 
Table 4.2: Number of positive samples and total number of samples (from all sampling methods) in the AMI Building for the October 25-November 8 sampling period, by region of the first floor. The "Entry, atrium, bathrooms" region comprises all of the first floor that it not included in one of the other regions. The table is modified from Traeger et al. (2002).

\begin{tabular}{|l|r|r|r|}
\hline & $\begin{array}{r}\text { Positive } / \\
\text { Total Samples }\end{array}$ & $\begin{array}{r}\text { Area } \\
\left(\mathrm{m}^{2}\right)\end{array}$ & $\begin{array}{r}\text { Fraction of } \\
\text { footprint area }\end{array}$ \\
\hline Mail suite & $7 / 10=70 \%$ & 75 & 0.03 \\
Mailroom quadrant (excl. mail suite) & $16 / 33=49 \%$ & 300 & 0.13 \\
Central offices and hallway & $17 / 61=28 \%$ & 375 & 0.16 \\
Distant office quadrant & $11 / 43=26 \%$ & 300 & 0.13 \\
Entry, atrium, bathrooms & $4 / 17=24 \%$ & 300 & 0.13 \\
Photo library & $2 / 17=12 \%$ & 250 & 0.11 \\
Text library & $3 / 33=9 \%$ & 175 & 0.07 \\
Construction Area & $0 / 2=0 \%$ & 550 & 0.24 \\
\hline
\end{tabular}

Table 4.1 shows the number of samples that tested positive for anthrax, and the total number of samples, in various locations. The AMI building is a 3-story office building with 6300 square meters (68,000 square feet) of floor area. The index case's office was on the third floor; all other measurements tabulated were on the first floor. We use the term "mail suite" to refer to the mailroom and the adjacent areas that share the same two entry doors. The data suggest that the index patient did not, in fact, open an envelope containing anthrax at his workstation: the fraction of positive samples in the index patient's work area was not elevated compared to the rest of the building. The fraction of positive samples in the index patient's work area was far lower than the fraction of positive samples in the mailroom and the office of the mail sorter.

Figure 4.1 is a map of the first floor of the building, with the locations of positive and negative anthrax samples shown. Overall, there were 70 positive samples and 160 negative samples, and $30 \%$ of the samples on this floor were positive for anthrax. Even ignoring a construction area that occupied about $25 \%$ of the floor area and that was very sparsely sampled, there was some variation in the spatial density of sampling: the photo library had about half as many samples per square meter as the mailroom, for instance.

The "construction area" was sealed off from the rest of the building prior to the putative date of the anthrax releases, and was barely sampled. Samples in the rest of the building can be summarized as follows: samples in the mail suite (the putative release area) had a $70 \%$ chance of being positive; samples in the immediately adjacent offices had a $50 \%$ chance of being positive; samples in the photo library and the text library had about a 10\% chance of being positive; and samples elsewhere in the building, regardless of distance from the source, had about a $25 \%$ chance of being positive. Results are presented in detail in Table 4.2.

As Table 4.2 and Figure 4.1 show, the spatial distribution of positive samples outside the mail suite, which we believe is the release area, is neither uniform nor determined by distance from the mail suite. The "distant office quadrant" had about as high a fraction of positive samples as did the entry and atrium area, whereas the photo library and text 
library had low fractions of positive samples.

\subsection{Other Data on the Spatial and Temporal Spread of Contamination}

Agranovski et al. (2005) used Bacillus thuringiensis, which they claim is genotypically and phenotypically very similar to B. anthracis, as an anthrax simulant in envelopes. They placed 50mg in an envelope and opened it. The resulting size distribution in air had a peak near $2.5 \mu \mathrm{m}$ diameter, with about $65 \%$ of particles between 1.75 and 3.5 $\mu \mathrm{m}$. Airborne concentration at $0.1 \mathrm{~m}$ was about $50,000 \mathrm{CFU} / \mathrm{L}$ at $10 \mathrm{~cm}$ from the release. The particle cloud reached $1 \mathrm{~m}$ from release point in about $25 \mathrm{~s}$; this value is somewhat dependent on airflow conditions in the experimental chamber, which was quiescent, and would be different in a space with stronger airflow. A sphere with $10 \mathrm{~cm}$ radius has volume of about $4 \times 10^{-3} \mathrm{~m}$, so the observed concentration at $10 \mathrm{~cm}$ implies a total release of about $10^{7} \mathrm{CFU}$. Measured particle deposition in the Daschle suite suggests that the release was about thirty times larger than that, which is not surprising since merely opening the envelope (as was done in the experiments) would be expected to aerosolize much less agent than removing from an envelope a letter that has anthrax folded inside it or sticking to it.

Griffith et al. (2006) performed particle release experiments in a two-story building, and measured the concentration of deposited particle mass at hundreds of locations in the building. Figure 4.5 shows the results from one of the releases. The release was in the square room in the middle of the left side of the building, roughly in the center of the room. Air flow measurements made before the release found that air was flowing out of the open doorway, into the hallway, at about $0.3-0.4 \mathrm{~m} / \mathrm{sec}$. As the figure shows, most of the mass deposited within about fifteen meters of the doorway. The importance of air flow is clearly seen: most of the mass did not even deposit in the room where the release occurred, and very little of the mass moved down the hallway (towards the bottom of the map).

There is an important distinction between mapping particle mass and mapping particle numbers. A $10 \mu \mathrm{m}$ particle has 1000 times as much mass as a $1 \mu \mathrm{m}$ particle of the same material, so even if most of the particles are small, most of the mass is in the larger particles. The deposition rate is much higher for large particles than for small ones it is about 15 times higher for $10 \mu \mathrm{m}$ particles than for $1 \mu \mathrm{m}$ particles, for example, and most $10 \mu \mathrm{m}$ particles will deposit within 8 minutes — so a map of the deposited mass will look very different from a map of deposited particles. In the case of the experiments of Griffith et al. (2006), the particle diameters were roughly lognormally distributed with a geometric mean around $1.6 \mu \mathrm{m}$; more than half of the mass was in particles of between 5 and $20 \mu \mathrm{m}$ diameter. Almost certainly, most of the large particles deposited fairly near the release point, and these large particles are the main constituents of the concentrated deposition at the upper end of the hallway, while the lighter particles, with their much longer suspension times, traveled much farther. 


\subsection{Rough prediction of surface concentrations}

To understand the phenomena that control the gross features of the spatial contaminant distribution, and to very roughly quantify the surface concentrations that might be expected from a release of anthrax spores, we created a simple model of a single HVAC zone, as shown in Figure 4.6. The model as a whole could represent an entire building that is served by a single HVAC system, or (more typically) a portion of a building. We assumed that the region where the release occurred was connected to the rest of the HVAC zone only through the HVAC system, as would occur if the release occurred in an office with the door closed.

We assigned air flow rates and filter efficiencies to match typical real-world conditions, and predicted the particle fate (Figure 4.6 and 4.6) for various operating conditions and filter types. Results suggest that the filter type has only modest influence on the amount of material that deposits indoors: we predict about a factor of two difference (in the amount of material that deposits indoors) between typical filters and very good filters, not enough to make a difference in any plausible sampling- or decontamination-related decision.

The predicted spatial density in the release region (Region 1) is shown in Figure 4.6, relative to the spatial density in the initially uncontaminated region. The model implicitly assumes that the spatial distribution within a given region is uniform. The predicted spatial density of particles on the HVAC filter and on the floor of the HVAC return ducts are also shown. Results from Sippola and Nazaroff (2003) were used to predict deposition in the ducts.

The model predicts that the spatial density of small particles $(1-3 \mu \mathrm{m})$ will be about 10 to 100 times higher in the release region than in the initially uncontaminated region, and that for larger particles the disparity will grow to a factor of 1000 or more because larger, heavier particles deposit much more quickly. Since most real-world releases involve a range of particle sizes, most are expected to have surface concentrations near the release point that are 100 to 10,000 times higher than in distant areas of the same HVAC zone. This finding agrees with the experimental results of Sajo et al. (2002) and Griffith et al. (2006), although the experimental conditions in those cases differed somewhat from those that we modeled: they performed the release in rooms with open rather than closed doors, so in addition to HVAC operation the experiments were subject to direct airflow between zones.

The essential result from the experiments and the modeling is that in the event of a release of anthrax spores from a single location, the surface density of particles near that location will be around 1000 times higher (within about a factor of 10 either way) than elsewhere in the same HVAC zone. This is important because it means that if a release occurs in a building, there might be a very large portion of the building in which the release is hard to find (in the sense that each sample from that region has a very low probability of a positive result), while in the release region each sample has a very high probability of a positive result. 


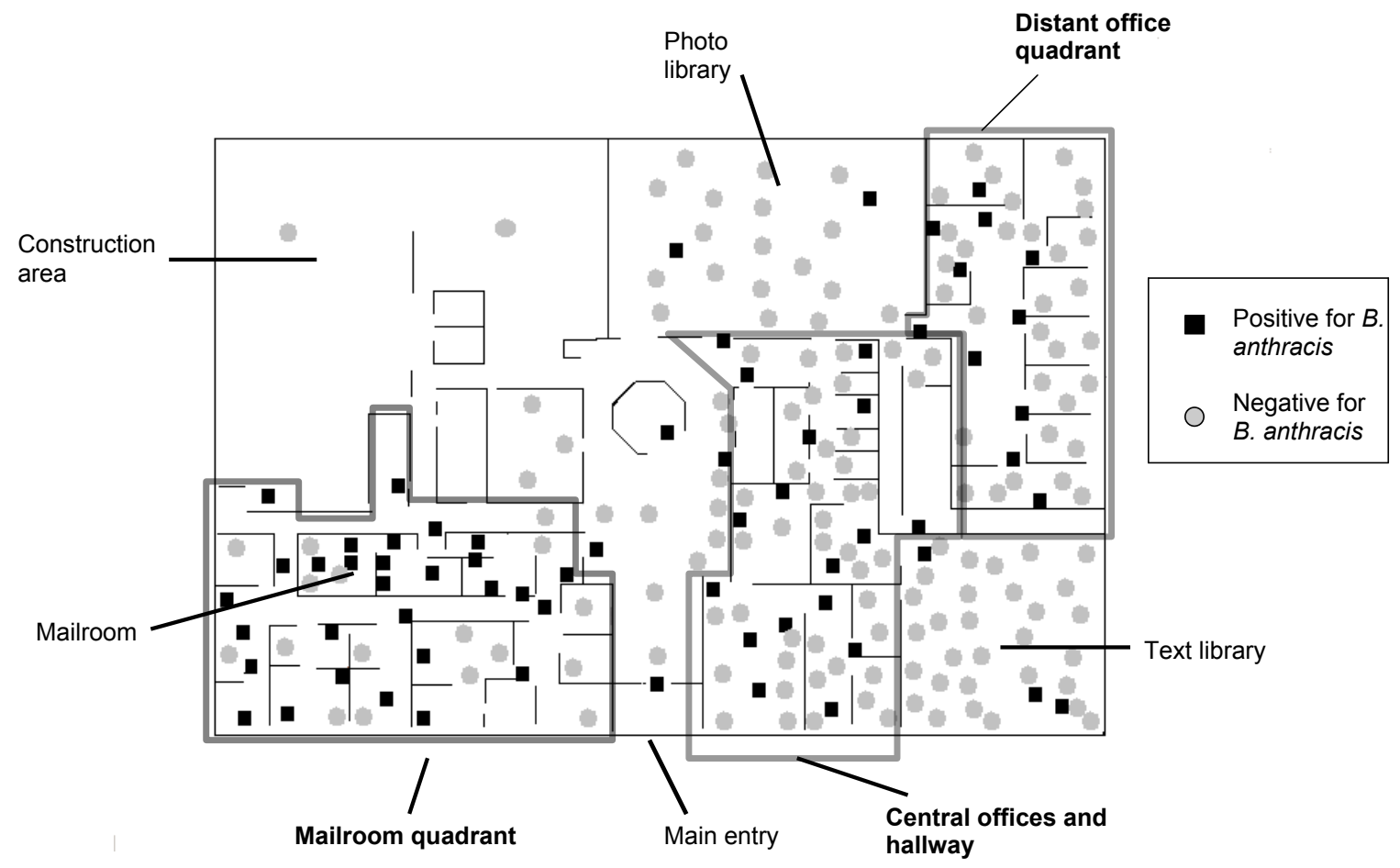

Figure 4.1: Map of the first floor of the AMI building, with positive and negative anthrax samples shown. From Traeger et al. (2002). 


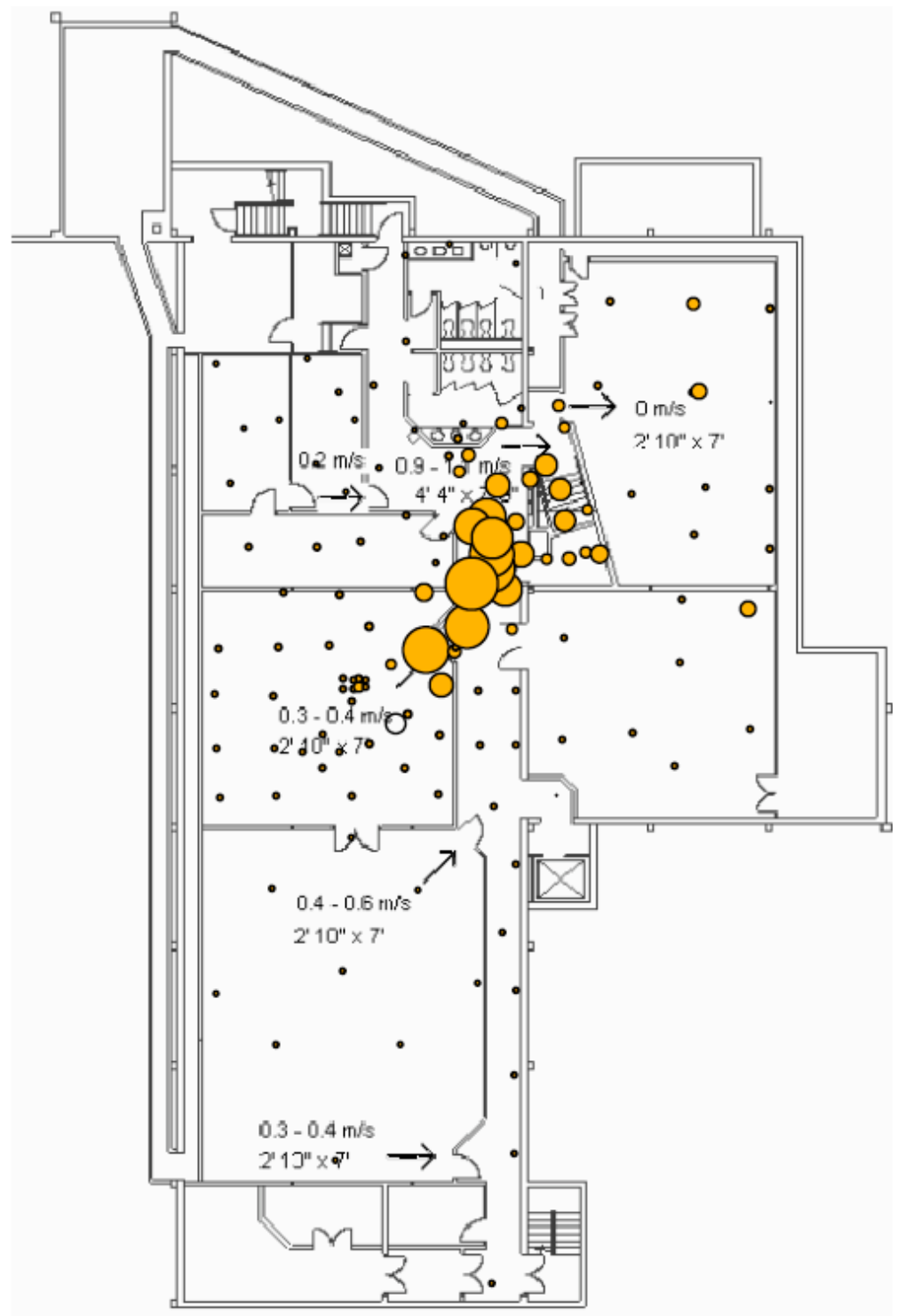

Figure 4.2: Deposited mass at various measurement locations in a particle release experiment. The measured mass is proportional to the cube root of the radius shown on the plot. (Imagine that each point represents a sphere, with the volume of the sphere being proportional to the mass measured at the location.) We used a cube root rather than a more common square root transformation because the square root led to such a large difference in size between the dots that either the small dots were too small to see, or the large dots covered huge areas of the plot. 


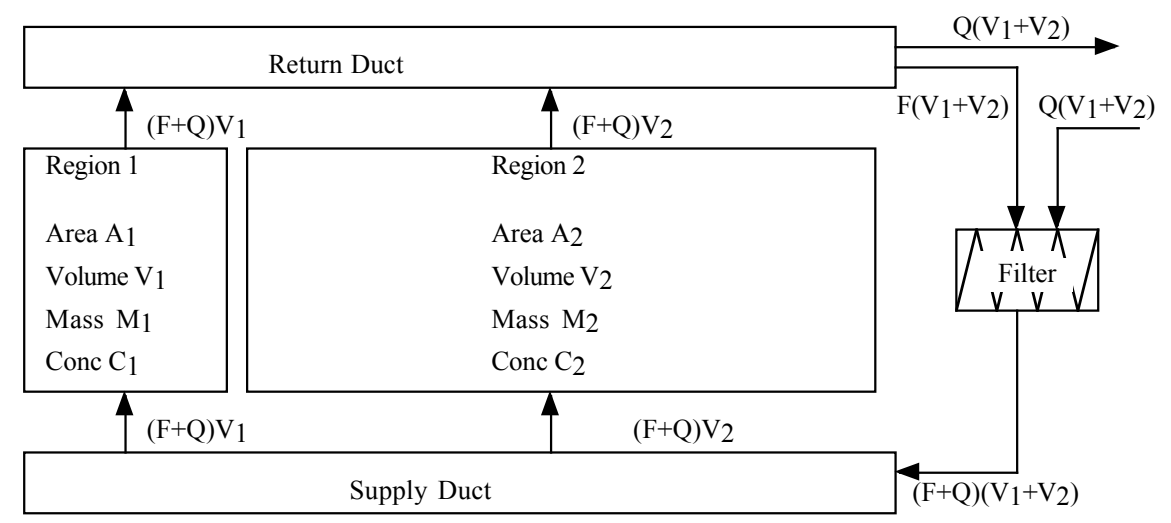

Figure 4.3: Schematic of the model building. The air in Region 1 is initially contaminated with a uniform density of monodisperse particles. The volumetric flow rate is shown for each flow path. $A_{1}$ and $A_{2}$ are the floor area of initially contaminated and uncontaminated portions of the HVAC zone $\mathrm{m}^{2}, V_{1}$ and $V_{2}$ are the volumes of those regions, $F$ is the recirculation rate through HVAC filters $\left(h^{-1}\right)$, and $Q$ is the indoor-outdoor air change rate $\left(h^{-1}\right)$.
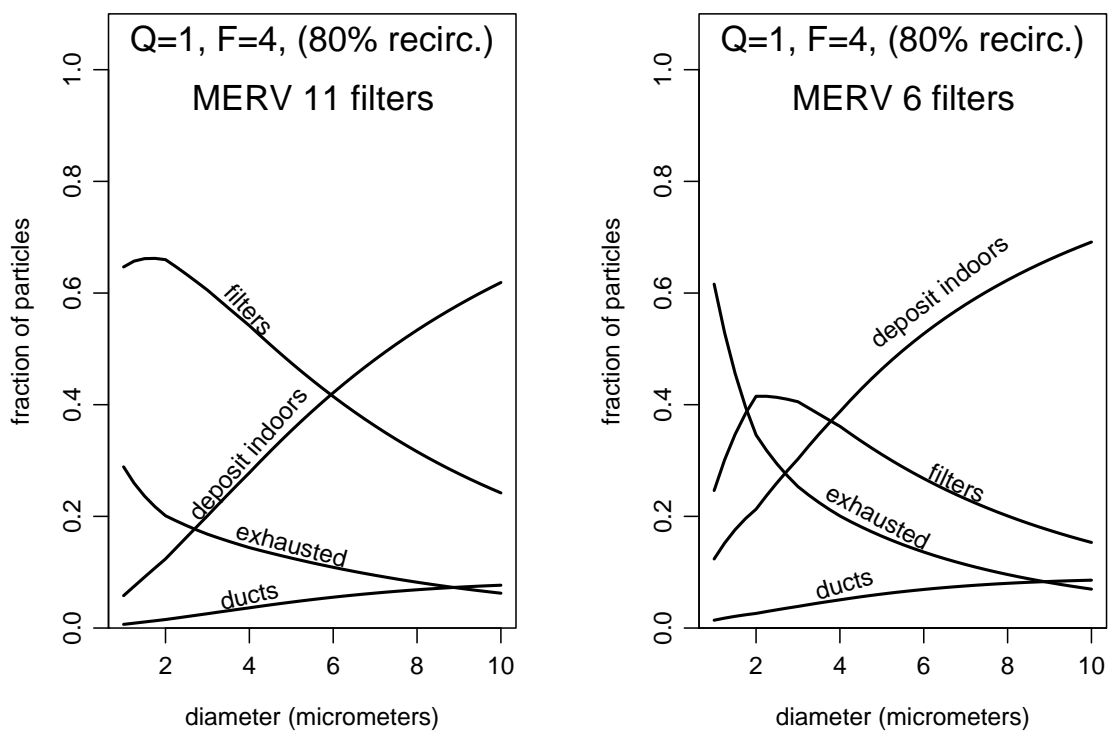

Figure 4.4: Particle fate (at $t \rightarrow \infty$ ) for MERV 11 and MERV 6 filtration, with 5 total air changes per hour and $80 \%$ recirculation. 

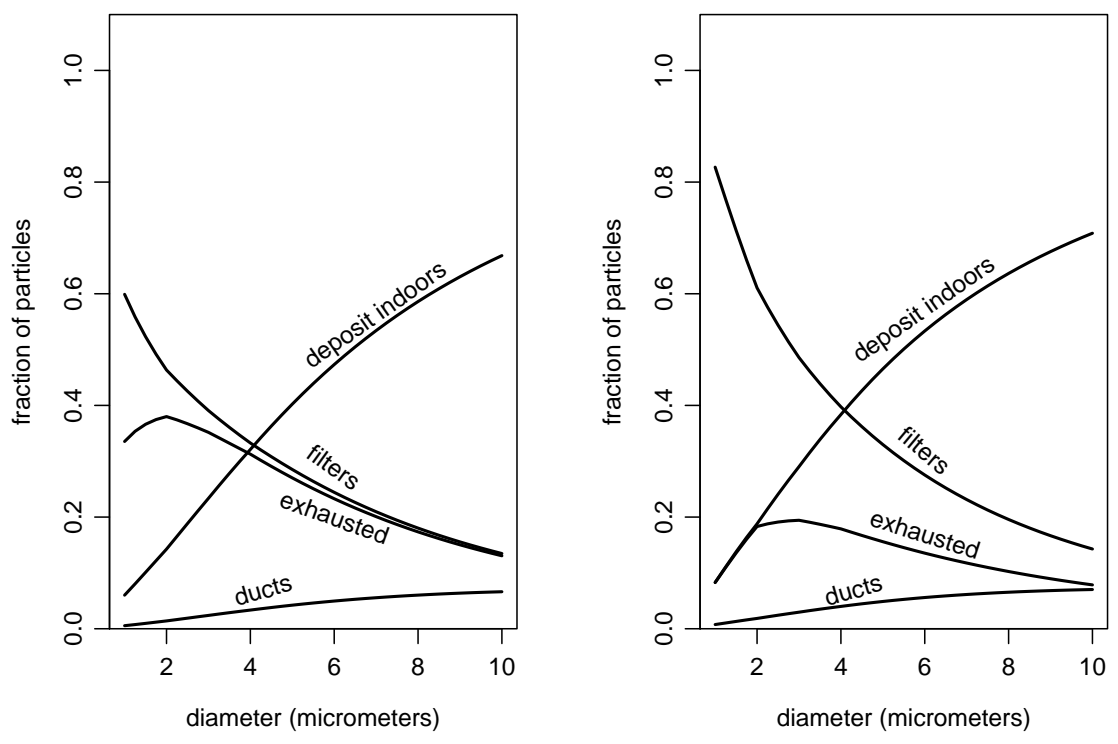

Figure 4.5: Particle fate (at $t \rightarrow \infty$ ) for MERV 11 (left plot) and MERV 6 filtration (right plot), with 4 total air changes per hour and $50 \%$ recirculation.

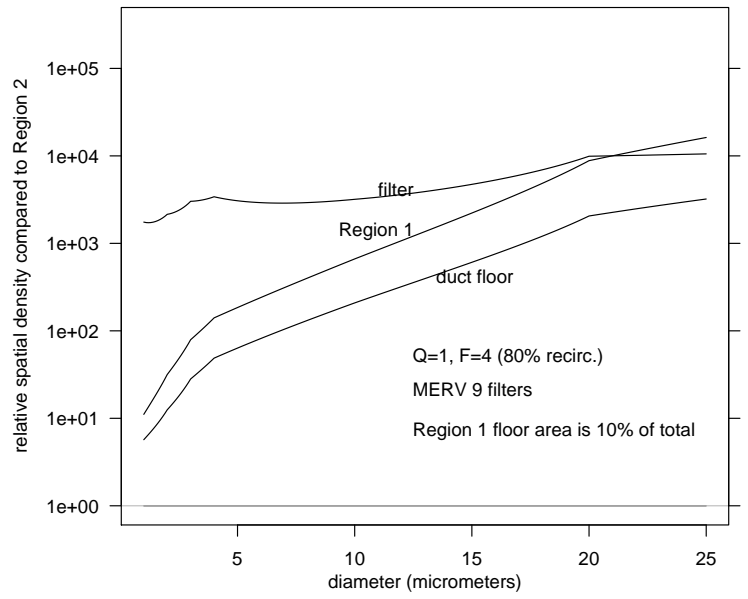

Figure 4.6: Final spatial density of particles relative to the initially uncontaminated region, for the case that the initially contaminated region is $10 \%$ of the total building volume. 

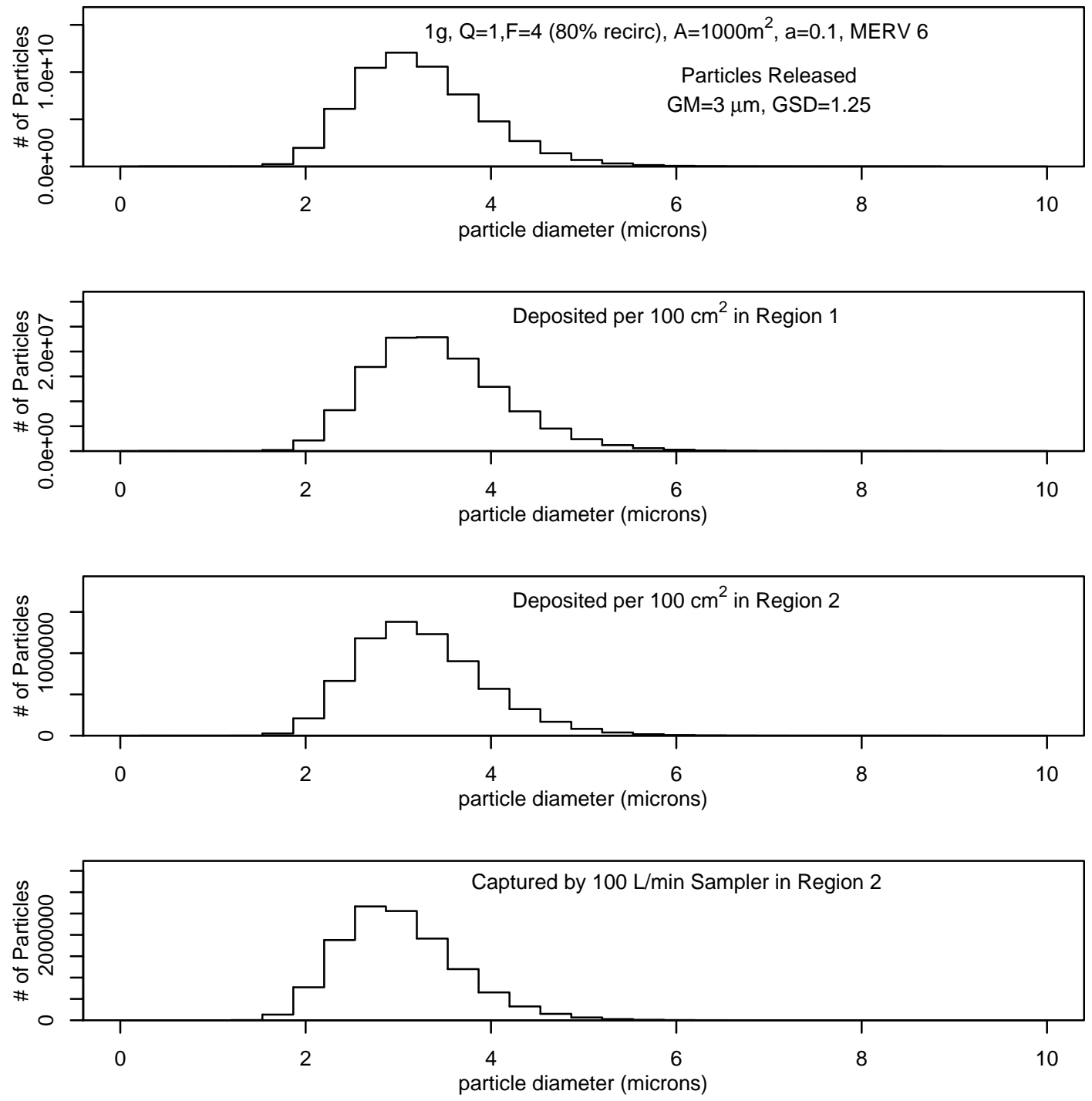

Figure 4.7: Final number of particles deposited or captured in various locations, as a function of particle size, for a polydisperse release. Region 1, the initially contaminated region, is $10 \%$ of building volume. The $1 \mathrm{~g}$ release has a geometric mean particle diameter of $3 \mu \mathrm{m}$ and a geometric standard deviation of 1.25 . 


\section{Chapter 5}

\section{Sampling}

One of the first responses to a suspected anthrax release will be to take samples to confirm that a release has occurred. Once it is determined that a release has occurred, samples will be taken to characterize the release. It is necessary to determine the strain of anthrax, and especially the amount and spatial extent of the contamination, in order to determine what decontamination measures should be taken.

In this chapter we discuss the sensitivity of current anthrax sampling methods, and implications for surface sampling. We also discuss the goals of characterization sampling, and suggest ways of determining the appropriate number of characterization samples. Characterization is a big task: "Characterization sampling involved collecting more samples at hot spots ... Characterizing the buildings represented a large portion of the time needed for response." (EPA 2002, p. 58).

\subsection{Detection Technologies and Effectiveness}

There are five main approaches to collecting surface samples: Wet wipe, vacuum, wet swab, dry wipe, and dry swab. They are arranged here in the approximate order of sampling effectiveness, from best (wet wipe) to worst (dry swab), as determined by the many studies on the subject (see Brown et al. 2007; Buttner et al. 2002, 2004a and 2004b; Farnsworth et al. 2006; Rose et al. 2004; and Sanderson et al. 2002). We say that the order listed above is in the "approximate" order of sampling effectiveness because details of the sampling protocols affect the results reported in these studies. Skolnick and Hamilton (2004) identified thirteen separate issues that may have reduced sampling effectiveness in the 2001 anthrax response.

All of the studies agree that the dry swab method is the worst, but some have claimed that a filter sock on a HEPA vacuum results in better results than wet wipes meaning, a more likely positive result when anthrax is present, and/or a better estimate of the surface concentration of anthrax - while others have claimed the reverse. The differences might be attributable to the various protocols that have been used, especially for vacuum sampling: simply vacuuming a much larger surface area, which is very easy to do, will collect many more spores but also more dust. The optimal area for vacuum sampling is not known. 
Table 5.1: Table adapted from Sanderson et al. (2004). Results from sampling at Brentwood postal facility, 23-28 October and 17-20 December, 2001.

\begin{tabular}{|l|rr|rr|rr|}
\hline Location & Wet Swab & Wet Wipe & \multicolumn{2}{|c|}{ HEPA } \\
& & & & vacuum sock \\
& N & \% pos & N & \% pos & N & $\%$ pos \\
\hline DBCS machine 17 & 13 & 93 & 19 & 100 & 8 & 100 \\
Other DBCS machines & 10 & 20 & 54 & 22 & 14 & 64 \\
Within 15m of DBCS 17 & 0 & 0 & 9 & 11 & 3 & 100 \\
Secure area 23m from DBCS 17 & 18 & 72 & 18 & 94 & 18 & 100 \\
Loading dock and transport office & 0 & 0 & 11 & 0 & 4 & 50 \\
Express mail room & 0 & 0 & 3 & 0 & 2 & 50 \\
Government mail area & 1 & 0 & 17 & 6 & 9 & 100 \\
Other locations in mail processing area & 3 & 0 & 20 & 0 & 15 & 67 \\
Administration and customer areas & 0 & 0 & 10 & 0 & 5 & 0 \\
\hline
\end{tabular}

Buttner et al. (2001) compared various surface sampling methods and compared Quantitative Polymerase Chain Reaction (QPCR) to culture methods for analyzing the resulting samples. They found that QPCR was slightly more sensitive than the standard culture method for testing for anthrax, and that wet wipe samples of vinyl tile yielded slightly higher counts than wet wipes from carpet. The very slightly higher efficiency of QPCR over culturing is probably not large enough to make a practical difference, and PCR has the drawback of not determining the viability of the anthrax (it does not distinguish between living and dead spores), but QPCR may be preferred to culture methods on other grounds such as faster throughput.

In a separate study, Buttner et al. (2004b) estimated a lower detection limit of approximately $40 \mathrm{CFU}$ per $\mathrm{m}^{2}$ for wet sampling and $100 \mathrm{CFU}$ per $\mathrm{m}^{2}$ for dry sampling, for detecting Bacillus atrophaeus spores via culture in an experimental situation, using a commercially available kit (known as BiSKit) that is used to sample a full square meter of surface. They did not define what they mean by "detection limit" — specifically, they do not suggest that there is a density threshold below which detection does not occur - but presumably they mean that spatial densities below this level will rarely result in a positive test. Buttner et al. also performed quantitative comparison of the number of CFU detected by culture and PCR, and found the detected numbers of CFU approximately comparable but with the culture technique having a statistically significant advantage for sampling a wood surface. They do not quote a sampling limit for PCR.

Table 5.1 shows results from co-located or approximately co-located samples in the Brentwood postal facility, from Sanderson et al. (2004). In this real-world test, the vacuum method appears to have performed best, in that it generated fewer "false negative" results. For example, HEPA sock samples from the carpet in the Brentwood transportation office tested positive (maximum $120 \mathrm{CFU}$ per gram of material), but the 3 wipe samples in this area were negative.

Brown et al. (2007) concluded that contamination must be at the level of a 100 CFU "per sampling unit" in order to have a high probability (which we assume to mean 


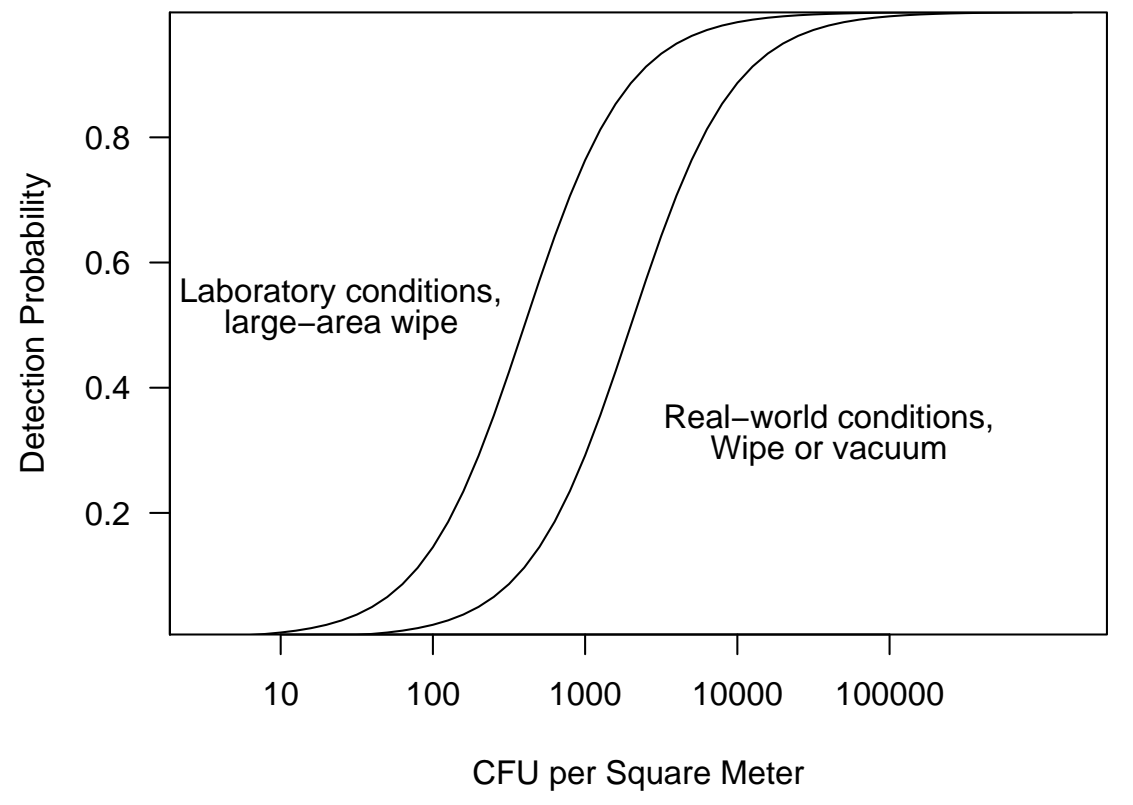

Figure 5.1: Probability of obtaining a "positive" result (y-axis) versus surface particle concentration (x-axis). The curve on the left summarizes approximately the results from laboratory experiments reported in Buttner et al. (2004b) and Brown et al. (2007); the curve on the right assumes that real-world sampling issues, such as rough surfaces and interference from other contaminants, will decrease sampling effectiveness. The curve on the right is in rough agreement with empirical results from the various wet wipe and vacuum sampling efforts in the 2001 anthrax responses.

$p>0.9$ ) of resulting in a positive sample, using wet wipes and current culture techniques, for a non-porous surface. Thus, for a wipe that samples only $100 \mathrm{~cm}^{2}$, detection is likely only if the surface is contaminated at $10^{4} \mathrm{CFU}$ per $\mathrm{m}^{2}$. However, if an entire meter is sampled then detection is likely if the surface is contaminated at $100 \mathrm{CFU}$ per $\mathrm{m}^{2}$, in good agreement with the findings of Buttner et al. (2004b). It is unknown whether conventional wipe methods, which typically have been used for $100-300 \mathrm{~cm}^{2}$, can readily be extended to areas 3 to 10 times larger, but it is known (Brown et al., 2007) that special kits can be used in this way.

These results are from laboratory experiments on steel (non-porous) and wood (moderately porous). Detection limits for other common materials, such as carpet or paper, are not known. For such materials - especially carpet - it is possible that a vacuum system would be more effective than wipe samples. Buttner et al. (2001) found wipe samples to be about as effective on carpet as on tile, but they did not investigate vacuum samples.

Using a logistic model for the variation of detection probability versus concentration, 
Figure 5.1 shows the approximate current situation as described in the literature.

Snyder et al. (2002) describe testing methods to confirm the presence of viable anthrax. Screening methods take a few hours; confirmation takes 12 hours or more. The Centers for Disease Control "Anthrax Q \& A" website (CDC, 2007) says that test results for anthrax "are usually available $24-48$ hours after the sample is received by the laboratory."

\subsubsection{Implications of Detection Probabilities}

\section{Simple case: uniform detection probabilities}

Ignoring for the moment the spatial variability in the probability of obtaining a positive sample, we consider the question of how many samples would be needed in order to be fairly sure of obtaining at least one positive.

If $N$ samples are taken, and if each sample has probability $p_{1}$ of being positive for anthrax, then the probability that they will all be negative is

$$
P(\text { all negative })=\left(1-p_{1}\right)^{N}
$$

so the probability of detecting contamination in the building - the probability of obtaining at least one positive sample - is:

$$
P(\text { at least one positive })=1-\left(1-p_{1}\right)^{N}
$$

$P$ (at least one positive) is plotted versus $N$, for several values of $p_{1}$, in Figure 5.2.

By solving Equation 5.2 for $N$, we can determine the number of samples that must be taken in order to attain a threshold probability $P_{T}$ of obtaining at least one positive sample:

$$
N=\frac{\ln \left(1-P_{T}\right)}{\ln \left(1-p_{1}\right)} .
$$

The number of samples $(N)$ required to have a probability $P_{T}$ of obtaining at least one positive sample is plotted in Figure 5.3 for several values of $P_{T}$. Twice as many samples are needed to attain a probability of 0.99 as are needed to attain a probability of 0.9 , and four times as many are needed to achieve a probability of 0.99 as are needed to attain a probability of 0.81 .

\section{More realistic case: variable detection probabilities.}

We next consider the more realistic case that different regions of a building have different probability that a single sample will be positive (we say different "regions" of a building rather than different "areas" to avoid later confusion with surface area and floor area). If there are two regions, with probability $p_{1}$ and $p_{2}$, and if $n_{1}$ samples are made in the first region and $n_{2}$ samples are made in the second, then:

$$
P(\text { at least one positive })=1-\left(1-p_{1}\right)^{n_{1}}\left(1-p_{2}\right)^{n_{2}}
$$

Figure 5.4 shows contour plots of the probability of obtaining at least one positive test, as a function of the number of samples in each region. Each contour forms a 


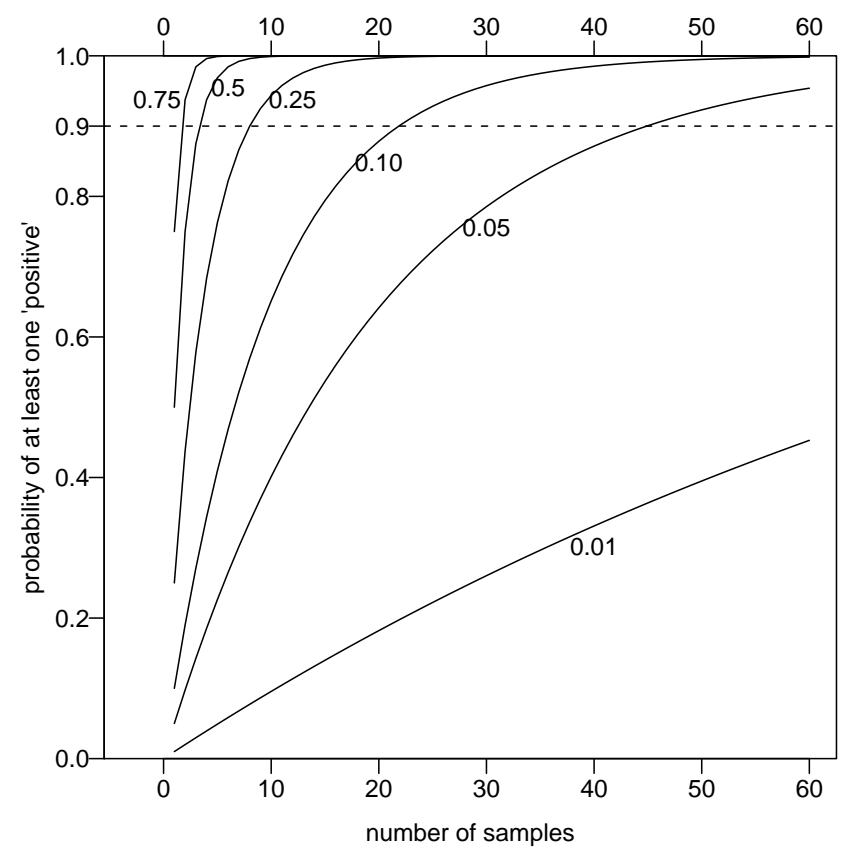

Figure 5.2: Probability of obtaining at least one 'positive' sample (y-axis) versus number of samples (x-axis). The probability that any single sample is 'positive' assumed to be either $0.01,0.05,0.1,0.5$, or 0.75 . The number of samples needed to ensure a given chance of obtaining at least one 'positive', such as a probability of 0.9 (dashed line), can be determined from the plot, or from Equation 5.3. 


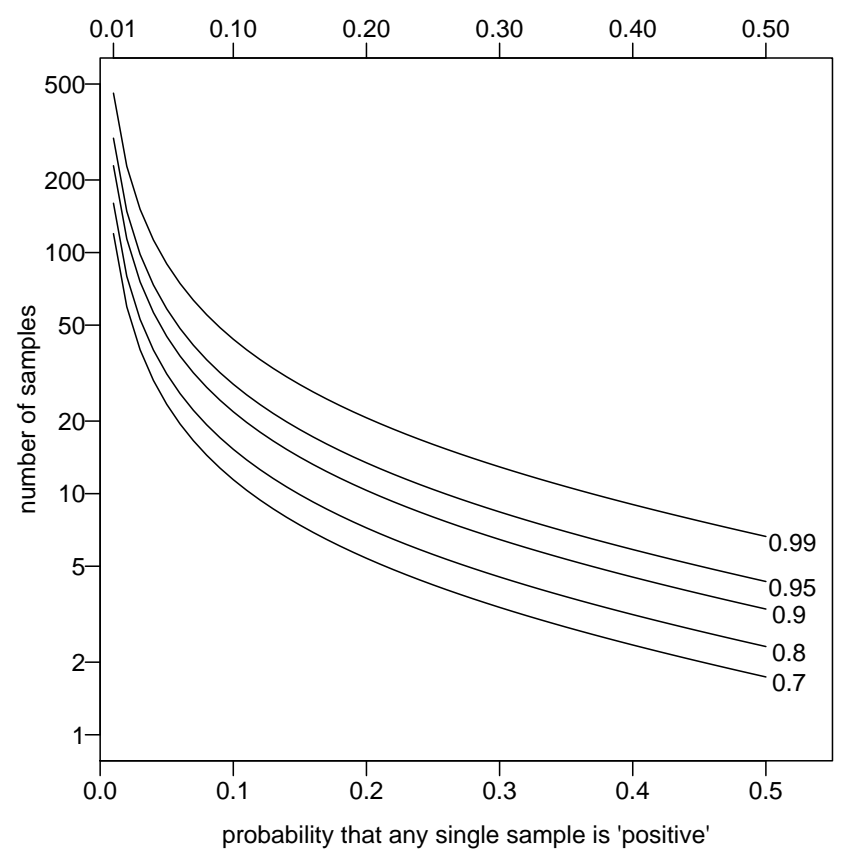

Figure 5.3: Number of samples necessary to attain a $0.7,0.8,0.9,0.95$, or 0.99 probability of obtaining at least one positive, versus probability that any single sample is positive. Y-axis is logarithmic.

straight line with slope $d n_{2} / d n_{1}=-\ln \left(1-p_{1}\right) / \ln \left(1-p_{2}\right)$. If $p_{1}>p_{2}$ and $p_{2} \ll 1$ then $d n_{2} / d n_{1} \approx-\left(p_{1} / p_{2}+p_{1}^{2} /\left(2 p_{2}\right)\right)$. If $p_{1} \gg p_{2}$ then it is much better to have a few additional samples from region 1 than to have a lot more additional samples from region 2. For example, if $p_{1}=1 / 2$ and $p_{2}=1 / 8$, then $d n_{2} / d n_{1} \approx-5$, so taking a single additional sample from region 1 would allow taking five fewer samples from region 2 without changing the probability of obtaining at least one positive sample.

More generally, with $\mathrm{m}$ regions:

$$
P(\text { at least one positive })=1-\Pi_{\mathrm{i}=1}^{\mathrm{m}}\left(1-\mathrm{p}_{1}\right)^{\mathrm{n}_{\mathrm{i}}}
$$

Figure 5.5 illustrates the benefits of making sure that the highly contaminated portion of a building is eventually sampled. We considered a case in which the probability that there is an $85 \%$ chance that a sample will be positive if it is taken in a heavily contaminated part of a building, and only a $5 \%$ chance if it is taken in a lightly contaminated area. The heavily contaminated area is assumed to occupy $1 / 20$ of the area of the building. The plot shows three curves, as a function of the number of samples taken: (1) probability of obtaining at least one positive sample if all samples are in the relatively uncontaminated portion of the building, (2) probability of obtaining at least one positive sample if samples are taken at random throughout the building, and (3) probability of obtaining at least one positive sample if sampling follows a grid pattern so that the heavily contaminated area is guaranteed to be sampled if the sample size is large enough. As the figure illustrates, if there is a large disparity between portions of 

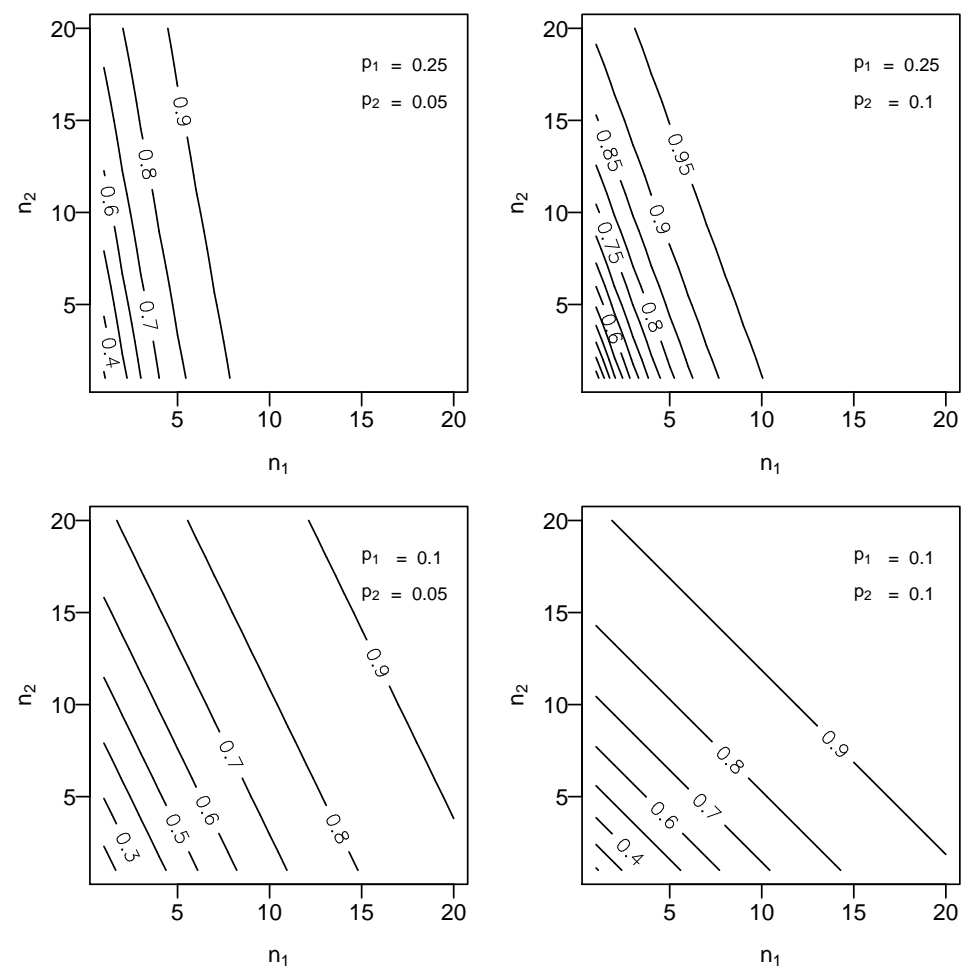

Figure 5.4: Contours indicate the probability of obtaining at least one positive sample, as a function of the number of samples taken in each of two different regions of the building. Each of the $n_{1}$ samples from one region has probability $p_{1}$ of testing positive, and each of the $n_{2}$ samples from the other region has probability $p_{2}$ of testing positive. Each plot shows a different combination of $p_{1}$ and $p_{2}$, noted in the upper right corner. 


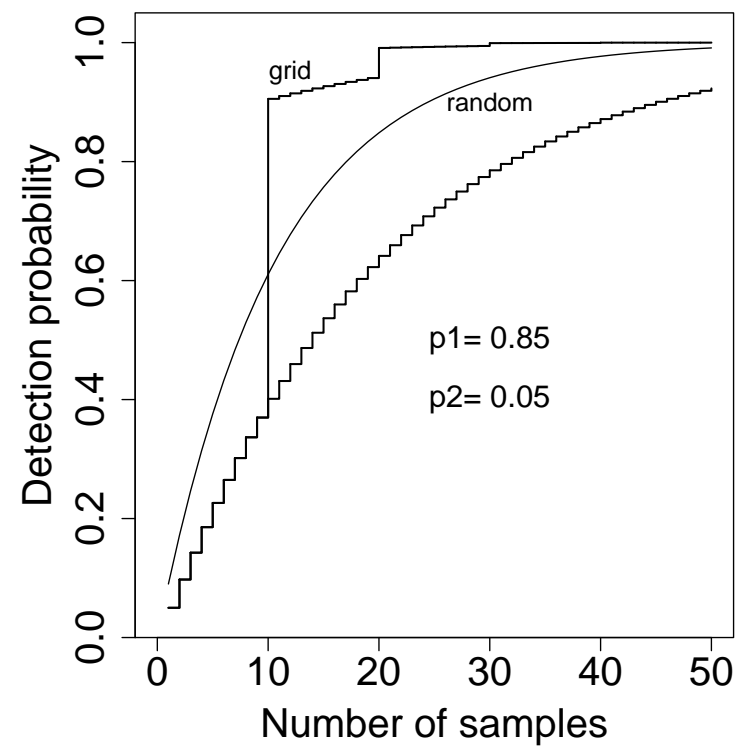

Figure 5.5: Probability of at least one "positive" sample (y-axis) versus number of samples (x-axis). The probability that a sample from the initially contaminated region ("Region 1") is 'positive' is assumed to be either $0.1,0.5$, or 0.9 . Region 1 is assumed to occupy $1 / 20$ of the building, and to first be sampled with the 10th sample, and at every 20 samples thereafter. The probability that a sample from Region 2 will be 'positive' is assumed to be $0.05,0.1,0.2$, or 0.4 times the probability in Region 1 (separate sub-plots).

the building in the probability that a sample will be positive, then it is very important to sample throughout the building.

\subsection{Number of Samples Required to Detect Contamination}

We can now attempt to determine the number of samples necessary in order to confirm that the level of contamination in a building or a region of a building is low enough that the risk of occupancy falls below a specified level. We do this as follows:

1. Decide on a level of risk that is acceptable.

2. Convert the risk to an airborne spore concentration, via an assumed dose-response curve.

3. Convert the airborne spore concentration to a surface concentration, using an assumed resuspension rate.

4. Convert the surface concentration to a probability that any single sample is positive for anthrax, using a sampling effectiveness curve.

5. Find the number of samples needed, for the single-sample probability determined in the previous step, such that if all the samples are negative then the building 
or region is "safe" with a specified certainty (such as 0.999 probability). (This step does not use the Bayesian approach of assigning a prior probability that the building is safe). A single positive sample is assumed to be enough to detect that the building or region is contaminated to an unacceptable level, although in practice confirmatory sampling should be performed of course.

Figure 5.6 allows the calculation of the necessary number of samples: it connects the risk from a 1-hour exposure to the number of samples that must be taken in order to confirm that a building or region is safe, with a specified level of certainty. It is an extension of Figure 3.3 and works the same way; in fact, the top two panels of Figure 5.6 are the same as Figure 3.3, slightly altered to allow the acceptable risk to be selected along the top of the plot rather than the bottom. The detection probability curves on the lower left panel are approximations to Buttner et al. (2004a) and Brown et al. (2007).

Figure 5.6 assumes the worst case, from the standpoint of detecting a very low level of contamination: it assumes that the building or region is uniformly contaminated (and that the resuspension rate is also uniform), so that the risk is equal everywhere. If the level of contamination were really uniform, it would not matter where the samples are taken: all of the samples could be taken in the same room, for example. The only factor that would lead to some samples being positive and others negative would be the statistics of the sampling procedure itself (the lower left panel of Figure 5.6): a very high surface concentration of spores will be detected nearly $100 \%$ of the time, and a very low concentration will be detected nearly $0 \%$ of the time, but there is an intermediate range over which each sample has some intermediate probability of returning a positive result. Thus, even if an region is uniformly contaminated, some (or many) samples may result in negative findings while others are positive.

In the more realistic case that the level of contamination is high in some regions and low in others, the average risk can be the same as in the uniform case but the number of samples required may be smaller. If the region near the release location is much more heavily contaminated than other regions, a grid sampling scheme or other system that caused samples to be taken near the release location will reduce the number of samples needed. In practice there is no advantage to taking multiple measurements in the same location, and a large potential advantage to sampling at spatially dispersed sites, so a grid sampling method should certainly be used. A computer program such as BROOM (Griffith et al. 2006) can help to create such a grid and to track the resulting data.

Even if there is a highly contaminated part of the building where the surface concentration of anthrax is higher so that it is readily detected, there might still be decisions - such as whether some other wing or floor of the building is adequately safe - that require sampling of a rather uniform, low concentration of spores. Figure 5.6 will still apply, outside the heavily contaminated region. The figure will also apply if anthrax is introduced to a building in a way that produces a rather uniform distribution of surface contamination, such as via an HVAC intake.

Attempting to be very "conservative" (i.e. highly protective) in all of the sub-decisions - the choice of an acceptable risk, the dose-response relationship, the resuspension rate, the assumed sampling effectiveness, and the level of certainty that a building is "safe enough" - will lead to infeasible or even ludicrous decisions. For example, choosing $10^{-8}$ as an acceptable risk per hour, and assuming high risk per dose and a high resuspension 
rate, will lead to the conclusion that even 0.1 spores per square meter is an unacceptable level of contamination. The probability of detecting such a low level of contamination cannot really be determined from the model that we apply (see the lower right panel of 3.3) since it requires extrapolation to surface concentrations far lower than those that the models are based on, but the probability of getting a positive sample from any single measurement at such low concentrations is probably less than $10^{-3}$. Thousands of measurements could be made in a building contaminated at this level, without necessarily finding a positive result.

If a risk level is chosen, Figure 3.3 can be used to determine the required number of samples to be sure the building is safe at that level. It can also be used in the reverse direction, to determine the level of risk that is implied to be acceptable if a given number of samples is taken. This could prove useful because the number of samples that can be processed may be a limiting factor. Heller et al. (2002) reported that the New York Public Health Laboratory increased its staffing by a factor of 40 and its laboratory space by a factor of 10 to handle the workload from the 2001 anthrax response, but that the maximum throughput obtained was only about 100 samples per 24-hour shift. Processes and equipment have probably been improved since then, but on the other hand the number of buildings that require sampling could be many times higher in a future incident. 


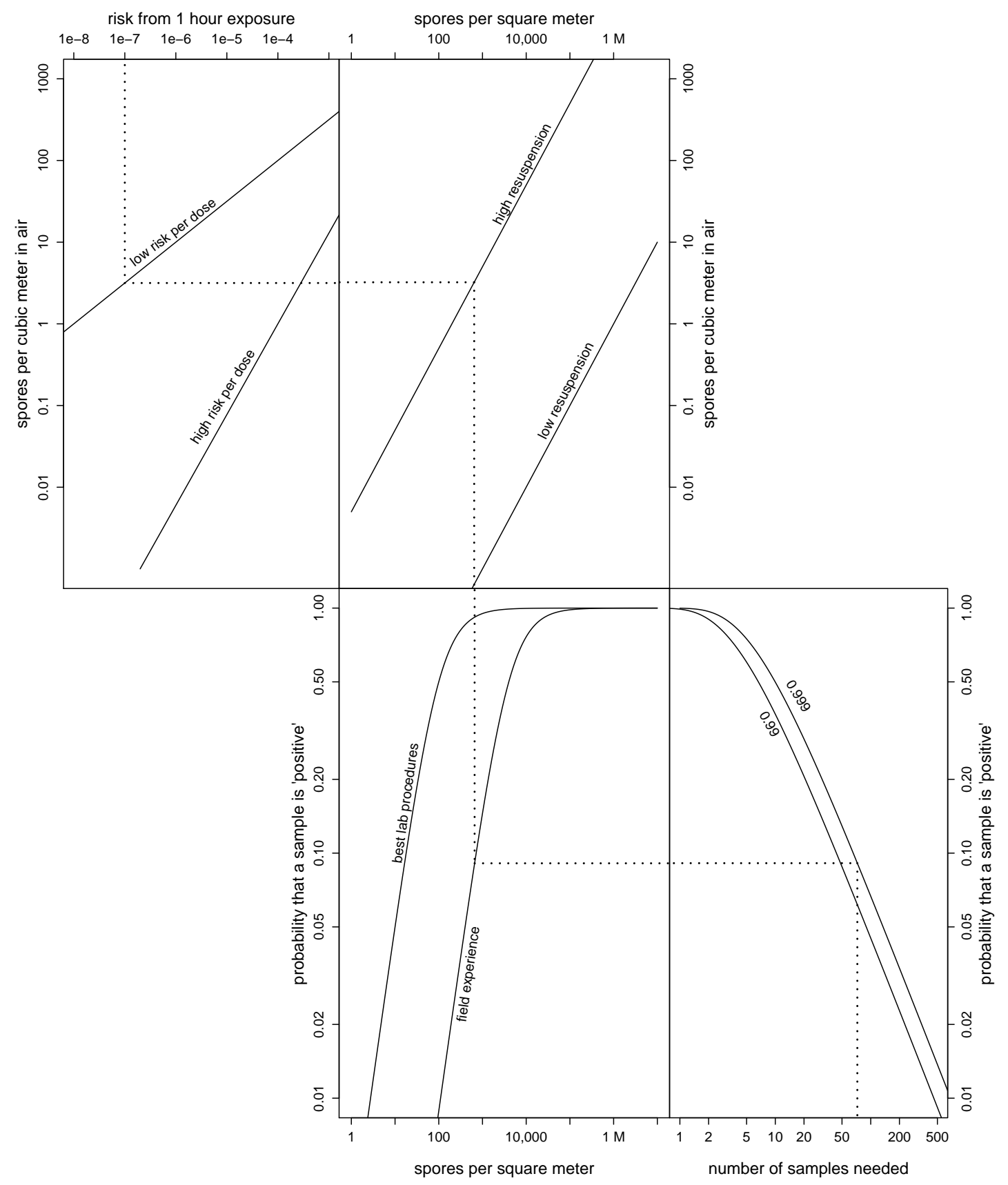

Figure 5.6: Determine the number of samples needed to ensure occupant safety: begin in the upper left portion of the figure by selecting an acceptable risk of infection for a person who will occupy the region for one hour. Read downward to a dose-response curve, then horizontally to the right to a "resuspension" curve. Continue downward into the plot below to a sampling effectiveness curve. Finally, read over to the plot at the bottom right, to a curve corresponding to probability of 0.99 or 0.999 of obtaining at least one positive sample. Read downward to the resulting $\mathrm{x}$-value to determine the number of samples needed (all of them negative) to attain the selected risk level. The dotted line on the figure shows an example. 


\subsection{Number of Samples Required to Characterize Contam- ination}

The previous section considered the number of samples required to detect contamination. In this section we discuss characterizing the contamination once it has been detected.

The goal of characterization is to affect decisions. The quantity and spatial extent of contamination will determine the type of decontamination that will be performed (local surface decontamination versus fumigation) and the physical size of the decontamination task (a single office, a wing of a building, an entire building). A key to controlling costs is to make sure that characterization focuses on those goals. For example, once a region has been determined to be heavily contaminated, there is no point to collecting more samples there.

The following are some rules of thumb to help select the number of characterization samples:

1. Begin by estimating the spatial variability of the contamination you are characterizing. If the contamination was brought in by air flow from a distant release, either directly or via the HVAC system, then the spatial distribution will be fairly uniform and there is no advantage to taking samples within a few meters of each other. If, on the other hand, the contamination was carried to specific locations (e.g. a contaminated object was carried through the building and put down at various spots) then a much higher sampling density might be required because some small areas might be much more heavily contaminated than their surroundings.

2. Once you have determined that an area (an office, a suite, a section of a building) is heavily contaminated, there may be no need to characterize the contamination in detail: you have already confirmed that the area needs a high degree of decontamination. You are probably better off taking fewer characterization samples and more samples to confirm decontamination effectiveness. See guidelines for post-decontamination confirmation.

3. Most important, remember that you will be doing post-decontamination confirmation. It is much more important to know that an area is safe after decontamination than to know exactly how unsafe it was before decontamination.

A key question for characterization is what level of spatial detail is needed concerning the spatial spread and quantitative variability of the contamination. The answer to this question is related to the decontamination methods that will be used and the practicalities of the decontamination.

\subsubsection{Hypothetical Situation 1: Small spots of contamination}

Suppose cross-contaminated mail is delivered to various offices in a building, and suppose that no article of mail is so contaminated that it releases a substantial number of airborne spores. In this case, contamination will be localized to spots that were touched by the contaminated mail, and perhaps spots that were touched by people who had recently handled the contaminated articles. Unfortunately, finding every spot of contamination 
would require an extremely intensive sampling scheme: enough samples would have to be taken to ensure that not a single contaminated spot is missed; the contaminated spots can then be decontaminated. In this hypothetical case there are therefore two basic options: (1) accept that some contamination may remain in the building, and that this will lead to some small risk to occupants, or (2) decontaminate every region of the building that contains some spots of contamination, without trying to identify all of the spots. In either of these cases, the number of characterization samples will be fairly small, as discussed below.

If the level of contamination implied by option (1) is acceptable, then local decontamination is in order. There is no need to fumigate the entire building and destroy its contents. Instead, material that may have come in contact with contaminated articles should be destroyed. This would probably include everything on desktops, including documents and books. Books on bookshelves and files in cabinets would be a more problematic call: if a book or file was open when contamination was transferred to it, and is then put away, it will present a danger the next time it is used. But sampling every page of every book, and every file in every file cabinet, is clearly unrealistic. The decision of whether to destroy or decontaminate books and files, or simply to leave them be, will rely on judgment about the probability of contamination, and characterization sampling is unable to resolve this issue.

At any rate, items on desktops should certainly be destroyed (or, if truly critically important, decontaminated). Floors and desktops should be decontaminated using surface decontamination methods as opposed to fumigation; see the Decontamination section for available methods. There is little role for characterization samples because, in this hypothetical scenario, even obtaining several negative samples from a single desktop will not provide assurance that there isn't a contaminated item on the desk somewhere.

\subsubsection{Hypothetical Situation 2: Fairly large region heavily contami- nated}

Consider the anthrax releases in the Hart or AMI buildings, as discussed previously in this report. In these cases, airborne spores settled throughout an office suite (Hart) and throughout the first floor of a building (AMI); direct non-airborne tracking of material was also important in the AMI case. Area-wide decontamination was clearly called for in both the Hart and AMI buildings, and the proper role of characterization sampling in these buildings was to decide what portion of the building needed decontamination. (In practice, in both buildings the characterization sampling went far beyond this requirement, and many samples were taken in areas that were already known to require decontamination).

Efficient use of characterization samples when a large area is known to be heavily contaminated will focus on the boundaries of the contamination. Repeated sampling of highly contaminated areas is wasteful, but samples in areas that may or may not be heavily contaminated can serve a useful purpose by helping to delineate the boundaries between different responses (such as fumigation in one area and surface decontamination in another). For example, if one floor of a building is known to be heavily contaminated but the status of other floors is unknown, few characterization samples should be taken 
on the contaminated floor but many samples should be taken on other floors to determine whether they require fumigation.

\subsection{Post-Decontamination Sampling}

\subsubsection{Experience}

Every decontamination effort, whether local or whole-building fumigation, uses postdecontamination sampling to confirm that decontamination was successful. Fumigation efforts also usually use "biological indicators" or coupons that are contaminated with a known level of contamination, placed in the building prior to fumigation, and tested afterwards to confirm that decontamination was successful. We briefly discuss two typical examples, below.

\section{Hart Building}

Since non-porous vertical surfaces rarely exhibited contamination (only two wall samples tested positive during the entire response), one wall and one composite screen sample were collected from vertical surfaces. Ultimately, over 400 verification samples were collected in the 3000-square-foot Daschle suite. (EPA 2002, p. 55).

In addition to verification samples of remaining contamination, spores strips were used to confirm that decontamination was effective. Two types of spore strips were used, with known concentrations of Bacillus subtilis spores ranging from $10^{4}$ through $10^{8}$, by factors of ten. Additional strips had various concentrations of other Bacillus species (specifically, B. stearothermophilus, B. thuringiensis, and B. cereus). The goal of fumigation was to reduce the number of viable spores by least a factor of more than 1 million, which would yield no viable spores on spore strips that initially contained $10^{6}$ spores.

After the initial fumigation, verification surface samples and analysis of spore strips were analyzed. The desired reduction in viable spores was found not to have been attained. EPA (2002, p. 90) says "it was later determined that the humidity was too low, thus making the chlorine dioxide gas less effective." The humidity was increased for a second fumigation, which was successful.

The number of spore strips used in the Daschle suite - one per square foot was vastly greater than necesssary. Fumigation effectiveness is expected to vary with location, but not at a scale of one foot, so there is little point to placing many spore strips within a few feet of each other. This has been acknowledged by the EPA, which suggests that a standard should be developed to determine the number of spore strips (EPA 2002, p. 90).

\section{Sterling Mail Facility}

The most detailed data we were able to find concerning post-decontamination sampling was for Department of State Annex 32 (the Sterling mail facility). This 70,000 square foot (7500 square meter) mail sorting facility was decontaminated using Vaporous Hydrogen Peroxide (VHP) in 2002. Ecology and Environment (2003) describes the post- 
decontamination sampling, and says "the sampling program was primarily based on obtaining representative sample data for solid surfaces and airspaces inside Annex 32." The facility had been divided into separate zones for decontamination, and post decontamination sampling was performed separately for each zone, in the sense that each zone had its own sampling objectives. Each zone was approximately 200, 000 cubic feet (6600 $\left.\mathrm{m}^{3}\right)$.

Both surface samples and air samples were collected. Surface sampling locations were a mix of random, "focused," "biased," and "optional". "Focused" sampling was used for individual objects that had been shown to be contaminated during pre-decontamination sampling. Since almost all such objects had been removed from the building before decontamination, focused sampling was used only for two light fixtures that remained in place. "Biased" sampling refers to objects or areas that were near the previous locations of contaminated objects before they were removed. "Optional" sampling refers to locations where focused and biased sampling did not occur and that also were not randomly selected, but where there were concerns that fumigation penetration might not have been sufficient. The sampling plan allowed up to 10 such samples per zone.

A grid sampling scheme was devised, based on $100 \mathrm{~m}^{2}$ grid cell. The approach was to sample $10 \%$ of the area of each grid cell, or about $10 \mathrm{~m}^{2}$ per sample. Surface sampling was performed using a HEPA vacuum with a dust collection sock. After initially using a sinuous stroke over the area to be sampled, the protocol was switched to straight lines to improve sampling speed. Special methods were adopted for dealing with ceiling tiles and other difficult situations. Approximately equal numbers of samples were taken on the ceilings as on the walls.

Air sampling included the use of leaf blowers to increase resuspension of spores. Visible dust clouds were noted in most zones. Dust overloading was a problem, as some of the air samplers shut down automatically when their filters became clogged.

The total number of surface samples taken per zone varied somewhat, with a low of 30 and a high of 67 , and a total of about 300. All samples were negative.

We are not aware of any studies that show the effectiveness of using a vacuum to sample the large areas - up to $10 \mathrm{~m}^{2}$ - used in these samples; most of the reported vacuum samples have sampled a few hundred $\mathrm{cm}^{2}$, or less than $1 \%$ of the area sampled in the Sterling samples. On the one hand, for a given level of surface contamination the number of spores collected should be approximately proportional to the area sampled, and so should be 100 times higher for these samples. On the other hand, the amount of dust collected will also be 100 times higher. Whether the probability of obtaining a positive result by collecting such a large sample is higher, lower, or the same as when collecting a smaller sample, is not known.

It is possible to use Figure 5.6 to see what level of risk is implied by these negative samples, or rather, to say that the risk is extremely likely to be less than some particular value. Considering the building as a whole, and assuming that we are interested in $99.9 \%$ certainty, we begin at the bottom left of the figure, at the location of 300 samples on the x-axis. (Since this axis is logarithmic, 300 samples is just a bit to the right of the "200 samples" mark). Go up to the 0.999 line, which we intersect at roughly $y=0.02$, meaning that if the probability of obtaining a sample were 0.02 (i.e. $2 \%$ ) then there is a $99.9 \%$ chance that we would have obtained at least one positive sample. 
From this point we move horizontally to the left to the "field experience line" on the adjacent plot. We intersect the "field experience" line at about $x=300$ spores per $\mathrm{m}^{2}$ meaning that at this surface spore density there is about a $2 \%$ chance of an individual sample being positive.

We now move straight upward onto the plot above, which relates this surface spore density to the expected airborne concentration of spores in an occupied building. For the Sterling Mail building, with no carpet and with much of the floor area undisturbed for a lot of the time, the "high resuspension" case will definitely not apply. Choosing a point between the "low resuspension" and "high resuspension" lines is appropriate. The situation in the building is realistically probably closer to the "low resuspension" case; a point that is visually midway in between the two curves is a reasonable choice that may be slightly "conservative" (i.e. protective). In our example, with about 300 spores per square meter on the ground, this gives us an estimate in the neighborhood of 0.03 spores per cubic meter in the air.

From that point, we move horizontally to the left, into the dose-response relationship portion of the plot. Here we see the enormous impact of the uncertainty in dose-response. If we assume that the risk for a given dose is near the high end of plausibility, then we are only claiming, with our 300 negative measurements, that we are $99.9 \%$ sure that the risk is less than $10^{-5}$ per person hour that an individual in the building will contract anthrax; considering the dozens of employees in the building, leading to hundreds of person-hours of exposure every day, this risk should surely be considered unacceptably high. In short, if we believe the risk per dose is very high, and that it may be uniformly contaminated, then even 300 negative samples should not convince us that the building is safe.

On the other hand, if the risk for a given dose is near the low end of plausibility, then we are $99.9 \%$ sure that the risk is less than about $10^{-9}$ per hour (well off the left edge of the plot). In this case, as much as a single anthrax case would be extremely unlikely even if the building were heavily occupied for a decade, even if we ignore the fact that the anthrax will lose potency over that period.

Having said that, it is worth raising two issues we have already discussed: First, the fact that anthrax is so rare, in spite of the hardiness of anthrax spores, suggests that the high risk-per-dose line substantially overestimates the risk from small numbers of spores. We have included the high risk-per-dose line only because a dose-response relationship like that one has been suggested by one researcher. Second, the relationship between acceptable risk and the number of negative samples is based on the assumption of uniform contamination of the building. If, instead, the building has "hot spots" where contamination is easily detectable, and "cool spots" where it is not, then the number of samples required to be sure of safety depends on the size of the hot spots rather than the statistics used in the plot. Specifically, in the Sterling facility 300 samples would be sufficient to take about one sample every 5 meters in a grid throughout the building, and if any of those grid locations had high anthrax concentrations (exceeding 10,000 spores per square meter or so) then detection would be very likely. In short, making highly protective choices in navigating Figure 5.6 will likely result in an extreme over-estimate of the actual risk; we recommend making "best guess" choices rather than "conservative" choices. 


\subsection{Recommendations}

The problem of post-decontamination sampling is similar in many ways to that of sampling to determine whether a building is contaminated: in both cases, the main goal is simply to determine whether the level of contamination in a building is acceptable, with a secondary goal of determining the approximate spatial extent and severity of contamination if it is present. As such, the section on "Number of Samples Required" in Chapter 4 is relevant for post-decontamination sampling as well. Specifically, Figure 5.6 will still apply.

However, there are two ways in which post-decontamination sampling differs from sampling to detect. The first is that the location of some "hot spots" - areas that were highly contaminated prior to decontamination - will already be known from characterization sampling. If the effectiveness of decontamination at these locations is typical of the effectiveness at other locations, then these hot spots represent the "worst case": areas that were initially highly contaminated. If the hot spots are successfully decontaminated by fumigation, then the rest of the building is sure to also be safe. A conservative (i.e. highly protective) strategy would be to not perform local surface decontamination on these areas, but instead to perform fumigation of the entire contaminated portion of the building until the hot spots are reduced to acceptable levels (perhaps undetectable levels) of contamination. Alternatively, the change in the number of viable spores can be determined by comparing quantitative estimates pre- and post-fumigation, to determine the kill rate. This would essentially treat a known hot spot as if it is a Biological Indicator or coupon. Once a kill rate is obtained that is considered sufficient for areas other than the hot spots, the hot spots themselves can be decontaminated using surface decontamination methods.

The other way in which post-decontamination sampling differs from sampling to detect contamination is that post-decontamination sampling can use information from Biological Indicators or coupons to gain confidence that decontamination was successful. If BI's and coupons indicate that a sufficiently high kill rate was achieved, then we have substantial prior information that the contamination level in the building should be low. From the perspective of Bayesian statistics (see Gelman et al., 1995, chapter 2) this knowledge or belief can be applied by use of a "prior distribution," which is mathematically equivalent to starting off with a certain number of negative samples, before the first actual sample is collected. For instance, if the coupons indicate that an $8 \mathrm{log}$ kill has been achieved (that is, the number of viable spores has been reduced by a factor of $10^{8}$ ) then we have substantial confidence that the level of contamination in the building has been reduced to a safe level, before we look at the first post-decontamination sample. That "substantial confidence" might be equivalent to having 40 or 80 negative samples. Determining the correct relationship between a particular log kill level and the resulting number of equivalent negative samples would require a research project; as far as we know, this relationship has not been worked out by anyone.

Taking all of the factors above into account, we recommend that if fumigation is to be performed:

1. At least a few "hot spots" should not be decontaminated with surface methods before fumigation. These hot spots can then be used to help evaluate fumiga- 
tion effectiveness. They can be decontaminated with surface methods after the evaluation, if fumigation is deemed successful.

2. Post-fumigation sampling should be performed at the locations of known "hot spots", and also at some other locations. There is no point in sampling at locations where the pre-fumigation surface concentration is known to have already been low, so samples should be concentrated in areas that were previously unsampled, undersampled, or found to have relatively high surface concentrations. The exception is that if there are areas where the effectiveness of fumigation is not trusted - because of distance from the injection point, proximity to a leak from a non-fumigated area, or a challenging surface for fumigation - those areas should also be sampled even if they did not have particularly high levels of pre-fumigation contamination.

3. Biological indicators (also known as coupons or spore strips) with known levels of contamination (of a simulant for anthrax) should be used. There is no need to place them closer together than the distance over which the fumigation effectiveness can change effectively; in most cases this will be at least several meters spacing, and perhaps more. Ideally the kill rate for the coupons should be slightly lower than the kill rate for spores deposited in the building; this can be achieved by using a simulant that is hardier (i.e. less susceptible to the fumigant) than anthrax, or perhaps by using a coupon that does not allow the fumigant to be fully effective. Development of coupons or simulants that can predictably fill these roles would be very helpful for further decontamination efforts. 


\section{Chapter 6}

\section{Decontamination}

\subsection{Decontamination Technologies}

Whitney et al. (2003) performed a literature survey and summarized quantitative results for sterilization of B. anthracis and simulants. Some of their key results are:

1. Chlorine dioxide gas: $100 \%$ kill of Bacillus atrophaeus (also known as B. subtilis var. Niger) after 1 hour of $30 \mathrm{mg} / \mathrm{L}$ at $80-85 \% \mathrm{RH}$ and 30C (Jeng and Woodworth, 1990a). 30 minutes of $6-7 \mathrm{mg} / \mathrm{L}, 20-40 \% \mathrm{RH}$ at $23 \mathrm{C}$ caused 5 -log reduction on Biologic Indicators. The same conditions, with pre-conditioning of the air with 70-75\% RH for a period (apparently 0.5 hours, though unclear in the description) before 15-minute exposure, caused 100\% kill on Biologic Indicators. (Jeng and Woodworth, 1990b).

2. Methylene bromide: $100 \%$ kill of B. anthracis after 24 hours at 3.4-3.9 g/L, material on sterile filter paper strips.

Science Applications International Corporation (SAIC, 2005) conducted a comprehensive review of approaches to performing decontamination of buildings and contents in the event of biological decontamination. They relied largely on Review of Decontamination Technologies for Biological and Chemical Warfare Agents (Mitretek, 2003) which provided an overview of potential remediation technologies and included a literature review, and stated that "other primary sources consulted were the DoD Wide Area Decontamination Study (Battelle, 1999), materials from the EPA Technology Innovation Office (TIO), and other readily available references." Technologies identified in the SAIC (2005) report or in other sources (Fitch et al., 2003) as being useful or potentially useful for decontamination following an anthrax release are discussed briefly in the following sections.

\subsubsection{Surface-Applied Technologies}

1. Hypochlorite, including "pH-Adjusted Sodium Hypochlorite," or ASH. Hypochlorite is a standard military decontaminant. Common household bleach is a $3 \%$ $6 \%$ solution of sodium hypochlorite. Hypochlorite solutions are cheap, easily available, and effective with 10- to 30-minute contact time. They are corrosive and a 
skin and eye irritant, and require an EPA crisis exemption for whole-building use. Hypochlorite solutions, including household bleach, work well on hard surfaces but poorly on porous surfaces, and their effectiveness is substantially reduced by dirt and mud. Ordinary hypochlorite is classified as hazardous waste, but ASH is not.

2. Aqueous hydrogen peroxide is used for disinfecting hard surfaces in several industries, and special building decontamination products are available.

3. Aqueous chlorine dioxide is used to treat drinking water. It is effective against anthrax on non-porous surfaces at 500ppm for 30 minutes, and is commercially available. It requires an EPA crisis exemption for use for building decontamination. It is normally generated on-site because aqueous chlorine dioxide will release gaseous chlorine dioxide, at levels that can be dangerously explosive.

4. HPO2 (Trademark); this is "enhanced" aqueous $\mathrm{H}_{2} \mathrm{O}_{2}$. No details available.

5. "Sandia Foam" and "Decon Green." These are mixtures of a mixture of hydroperoxide and hydroperoxycarbonate anions with other chemicals that are sprayed from a canister and create a foam. They are available under trade names from a few manufacturers. They are claimed to reduce anthrax contamination by a factor of $10^{6}$ when properly applied, but did not achieve this in response to the 2001 anthrax response; see the section below concerning "other anthrax decontamination experience."

6. CASCAD (Trademark) is a foam whose active ingredient is sodium dichloroisocyanurate, also known as "fichlor." It has a long use as a disinfectant in the food industry. The foam was designed for exterior applications on military equipment, but SAIC (2005) asserts that it is being evaluated for use in buildings; we were not able to find any results or reports of effectiveness in buildings.

7. L-Gel (Trademark) is an "inexpensive" product (approximately $\$ 1$ per $\mathrm{m}^{2}$ ) whose active ingredient is potassium peroxymonosulfate. As of 2006, it was not yet commercially available.

8. A proprietary agent called "Virkon S [Trademark], produced by Antec International (Sudbury, Suffolk, UK), has been used in Europe to disinfect cattle potentially exposed to anthrax, and in hospitals to sterilize equipment and surfaces against biological agents." USDA (2003) says Virkon S "is a buffered peroxymonosulfate compound modified with the addition of surfactants and organic acids. In $1 \%$ solution, it has a $\mathrm{pH}$ of 2.6 and thus would be effective as an acid disinfectant if this were its only mode of action. With the additional activity of the oxidizer, it can be expected to be effective over a broad spectrum of organisms."

\subsubsection{Gas- and Vapor-Phase Technologies}

1. Chlorine dioxide gas was used successfully in several buildings in 2001, including the Brentwood and Wallingford postal facilities and the Hart building. It is effective on porous surfaces, and decomposes rapidly. It is explosive under pressure and 
so cannot be shipped, but must be created on-site. It requires high relative humidity (over 65\%) for maximum effectiveness. Commercial generation systems exist, including trade names ClorDiSys and Sabre. The chlorine dioxide concentration and relative humidity can be monitored by remote sensors in real time, so that changes can be made to ensure that exposure guidelines and spatial distribution guidelines are met. Past experience and laboratory work using real anthrax spores have led to the development of known effective exposures for various surfaces.

2. Hydrogen peroxide vapor was used to decontaminate two federal mail facilities in 2001 and is readily commercially available. VHP and mVHP (which includes ammonia) have been used in the past for building decontamination. VHP degrades to oxygen and water, while mVHP also leaves residual ammonia.

3. Ethylene oxide is widely used in hospitals. It penetrates porous surfaces and can be used to decontaminate documents and small items. It is flammable, irritating, toxic, and a probable carcinogen, so it is not considered acceptable for wholebuilding fumigation. It was used in the 2001 responses to decontaminate small items, in a special chamber.

4. Paraformaldehyde is routinely used for disinfection in research laboratories and is effective against anthrax. It has been used for building decontamination by the U.S. military. It was used to decontaminate some mail processing equipment in 2001. It is a probable human carcinogen.

5. Ozone has been mentioned in the past as potentially useful for deactivating anthrax, but it was not investigated in the SAIC report. We were not able to find any published data that suggest that ozone would be effective for decontaminating buildings.

6. Methyl bromide is used for pest control (e.g. termites), and will kill anthrax at sufficient concentrations. It is ozone-depleting and toxic.

\subsection{Experience}

All of the decontamination efforts following the 2001 anthrax releases shared many procedures, no matter what decontamination methods they used:

1. Segregate building contents into several types, such as (1) critical for decontamination, (2) important, so decontaminate if possible, and (3) unimportant, so discard.

2. For contaminated areas where fumigation will not be performed, perform local decontamination using surface-applied materials.

3. For areas where fumigation will be performed, remove all furniture and all items. In most cases carpet was also removed.

4. For areas where fumigation will be performed, create barriers to prevent fumigant from exfiltrating outside the intended area. 
5. For areas where fumigation will be performed, place "biological indicators" (spore strips or coupons) that can be used to determine whether decontamination was successful.

6. Perform decontamination.

7. Perform confirmatory sampling.

8. Repeat decontamination if necessary, and re-sample.

EPA (2002) reports several "lessons learned" that might decrease costs in future responses. For example, they report that identifying, handling, and preserving items identified as "critical" was a very time-consuming task, with many items being handled multiple times. Reducing both the number of critical items and the amount of time spent handling each item were identified as possible major improvements. They suggest performing a cost-benefit analysis in future events, to determine which items are economical to remove and decontaminate.

A cost-benefit analysis could be very simple, since each item would not have to be analyzed separately. Instead, cost estimates could be made for bagging, removing, decontaminating, and returning different classes of items, such as small, non-porous items (coffee mugs, staplers, etc.); small electronic items (calculators, mobile phones etc.); computers; and so on. If the cost of decontaminating the item exceeds the cost of discarding it and replacing it, it can be discarded.

Beyond the common features shared by all of the decontamination efforts, each effort did have unique features. Some of the decontamination procedures are described below in more detail.

\subsubsection{Hart Building}

The anthrax-containing letter that was opened in Senator Daschle's mail area led to substantial contamination of surfaces throughout the 3000 square foot (320 square meter) office suite. Other areas of the building were also contaminated, mostly through tracking of anthrax spores and from cross-contaminated mail, but the surface densities of spores were much lower in other areas. The EPA cleaned the less-contaminated areas of the building with chlorine dioxide liquid, Sandia foam, and HEPA vacuum.

Senator Daschle's office suite was prepared by constructing isolation barriers to contain chlorine dioxide. Fumigation was carried out for more than 12 hours. Sodium bisulfate gas was then pumped into the suite to scrub the chlorine dioxide from the air: sodium bisulfate reacts with chlorine dioxide to form oxygen and an innocuous salt (Vogt and Sorenson, 2002). Three thousand test strips of surrogate spores were exposed during fumigation. Samples were taken following fumigation, and 9 of the 377 samples showed trace amounts of anthrax. We were not able to obtain the locations of the positive samples, but Sharp and Roberts (2006) state that "Fumigation was repeated to remove viable spores still detected in the heating and ventilation system."

The Hart building cleanup cost $\$ 20 \mathrm{M}$ according to Vogt and Sorenson (2002). The EPA and CDC noted that even after the cleanup "there is a possibility of residual viable

spores," since sampling would not be able to detect a very low concentration of spores 
(Vogt and Sorenson, 2002). The Government Accounting Office also noted (Rhodes, 2005) that "negative results do not necessarily mean that a facility is free from anthrax."

Overall, EPA spent about $\$ 27$ million to clean up anthrax contamination in the 7 contaminated buildings on Capitol Hill (NRC 2005, p. 66), so about $\$ 7 \mathrm{M}$ was spent on buildings other than the Hart building.

\subsubsection{Brentwood Postal Facility}

The cleanup job at Brentwood was extremely large: the building has 700,000 $\mathrm{ft}^{2}(75,000$ $\mathrm{m}^{2}$ ) of floor area; 100 dock doors; and 235 skylights. Contamination was widespread.

All movable items were removed and decontaminated using a bleach solution. Doors and skylights were sealed before decontamination. Chlorine dioxide gas was made on-site and pumped into the building through the HVAC system and through flexible piping that carried the gas throughout the building. Mail sorting equipment was run during the decontamination so that gas could reach all parts of the machines. The temperature was held at $24 \mathrm{C}$, and relative humidity at $75 \%$, since these conditions are known to cause a much higher kill rate than normal room conditions. Monitors throughout the building confirmed that gas levels reached $750 \mathrm{ppm}$. Gas was maintained in the building for 12 to 48 hours. Over 8000 spore test strips were used to monitor effectiveness. 1200 workers were required on-site during the decontamination process. (Sharp and Roberts, 2006).

"Chlorine dioxide was used to decontaminate the 700,000 $\mathrm{ft}^{2}$ Brentwood postal facility, which took 1 year at a cost of $\$ 130$ million" (Wein et al., 2005). This cost includes removing and disposing of removable items, not just sampling and fumigating the building.

\subsubsection{Sterling Mail Facility}

Department of State Annex 32, the Sterling mail facility, is a 70,000 $\mathrm{ft}^{2}\left(7500 \mathrm{~m}^{2}\right)$ mail sorting facility with 20 foot high ceilings. The building was emptied of all furniture, office items, sorting equipment, etc.; the floors, walls, and ceilings were wiped down with soapy water; and then the building was fumigated with Vaporous Hydrogen Peroxide (VHP).

Irreplaceable documents and a small number of diplomatic pouches, totaling 23,000 pounds of material, were treated in an ethylene oxide sterilization chamber and retained for use. Non-essential items were handled as follows (Buxton, 2005):

1. Metal items, 372,000 pounds, were treated in a sterilization chamber and returned for reuse or recycled.

2. 2234 pounds of batteries, and 325 fluorescent lights, were treated on-site with SporKlenz and then sampled to confirm that they were not contaminated. They were then sent for recycling.

3. 4300 pounds of paraformaldehyde hazardous waste and 195 gallons of flammable liquids were sent to hazardous waste incineration, and waste was buried in a Class C Chemical Waste Landfill. 
4. 155,000 pounds of small items such as paper, telephones, computers, office supplies, etc., were incinerated and the resulting waste was buried in a Subtitle D landfill.

5. 448,000 pounds of large non-structural facility items such as insulation, furniture, office structures, bagged waste, etc., were steam sterilized and sent to a Subtitle D landfill.

The total cost of removal, treatment, return for reuse or destruction, and facility cleaning prior to fumigation was $\$ 4.3$ million.

According to SAIC (2005), "Over 70,000 lb of flat mail was treated by irradiation." and "over 46,000 diplomatic mail pouches were sterilized using paraformaldehyde, using pre-constructed chambers set up in the SA-32 building for this purpose." It is not clear how these numbers reconcile with the statement by Buxton et al. that a "small number" of diplomatic mail pouches were sterilized, although we speculate that there were actually a small number of pouches that contained a total of 46,000 individual pieces of mail.

\subsubsection{Other anthrax decontamination experience}

"Using guidance developed by USAMRIID [U.S. Army Medical Research Institute of Infectious Diseases], Fort Dietrich, Maryland, the [EPA OSC Decontamination] team proceeded to decontaminate the [Boca Raton Main Post Office] using a specially prepared bleach solution that had been used successfully by USAMRIID. Five other post offices in the area that were contaminated were also decontaminated using the same procedure, given that the contamination was isolated to a small number of hard surfaces and machinery that were non-porous" (Barth et al., 2003).

The Trenton, New Jersey mail processing and distribution center was decontaminated in summer 2003 using the same basic techniques as used at Brentwood. (Sharp and Roberts, 2006).

Small, low-concentration anthrax contamination was decontaminated at several locations on Capitol Hill in 2001 using chlorine dioxide liquid, HEPA vacuum, and/or Sandia foam. These locations included the Supreme Court Building (chlorine dioxide liquid); the Ford Building bomb squad offices (chlorine dioxide liquid, HEPA vacuum, and Sandia foam); the Longworth building (chlorine dioxide liquid and HEPA vacuum); the P Street Warehouse (chlorine dioxide liquid and HEPA vacuum); the Russell Building (chlorine dioxide liquid and HEPA vacuum); and the Dirksen Building (HEPA vacuum and Sandia foam).

EPA (2002) says that the experience with the Sandia foam in the Ford Building and Dirksen Building was not entirely satisfactory: "The foam was difficult to apply and clean up, and left a yellowish stain on surfaces" and, more importantly, "following [a] round of decontamination, a number of verification samples tested positive for anthrax." The company that supplied the foam, EnviroFoam Technologies, Inc., claimed that the foam was not applied properly and provided further training in its application. Several weeks after the application in the Ford and Dirksen buildings, Sandia Foam was used for some vertical surfaces in the Hart building that required a longer contact time than chlorine dioxide liquid could provide. There is no report on whether staining and difficult clean-up were still problems in that case. 
Cleanup of the AMI building was delayed for several years by legal and financial problems. The AMI building cost "significantly less than $\$ 5$ million" to decontaminate (NRC 2005, p. 63). No details on this decontamination were available to us.

\subsection{Selecting a Decontamination Method}

Considerations for selecting a decontamination method include the following:

1. Certainty that decontamination will be successful.

2. Cost of equipment and materials.

3. Ability to monitor success.

4. Cost of transport and installation of systems (such as pipes and reactors), if necessary.

5. Personnel effort required, including training time.

6. Resulting damage to building or contents, through corrosion, bleaching, etc.

7. Safety of residue, if any.

This list of considerations will not change for future events, but the method or methods that best meet the criteria will depend on circumstances and will change as technology changes. Chlorine dioxide was chosen for most (but not all) of the fumigations following the 2001 anthrax releases, and that choice was justifiable both before and after the fact: the probability of success was high, the risk of residual contamination was low, and the equipment and materials costs were reasonable. Once the method had been used once, personnel were familiar with it, and later decontamination efforts could draw on that experience.

We had hoped to learn some useful quantitative information about costs, but this has not been possible because we have not been able to get cost break-downs of the various decontamination projects so as to enable an "apples-to-apples" comparison. For example, the standout in terms of costs seems to be the AMI building, for which a cost "much less than $\$ 5$ million" is reported for decontamination of a 70,000 square foot office building that had one heavily contaminated floor, and two other floors with much lower levels of contamination but with spatially dispersed positive samples. This contrasts with, for example, the Hart Building, in which most of the $\$ 20$ million decontamination cost went to decontaminating a single 3000-square-foot office suite. This contrast is misleading, though, because the $\$ 5$ million cost for the AMI building decontamination apparently includes only the cost of performing and confirming decontamination of the building per se, whereas the cost quoted for the Hart Building includes many more elements: the cost of characterization sampling; the cost of disposing of non-critical items; and the cost of identifying, handling, preserving, and decontaminating critical items. The cost per square foot of decontaminating the Daschle suite was also increased by the need to build barriers to prevent the fumigant from entering other offices. Without a breakdown of personnel time into different tasks, we cannot tell how much time and money was spent 
on installing and operating the fumigation equipment, and how much was spent on the other decontamination tasks.

Many decontamination costs will not vary from one agent to the next, and indeed, the cost of fumigation may not be the dominant cost of the decontamination effort. As discussed in a previous section, many procedures must be carried out no matter what decontamination method is selected, such as identifying and separating "critical" items, destroying and disposing of contaminated non-critical items, and so on. Lost work days, the loss or destruction of important but perhaps non-critical items, disruption, etc., may cost more than the decontamination per se.

The report "Compilation of Available Data on Building Decontamination Alternatives" (SAIC, 2005) summarizes the current state of knowledge concerning materials costs, effectiveness, and safety of all or most of the currently available products for bioagent decontamination. The report does not recommend any particular product, but two products stand out as being most suitable for building decontamination, given the current state of technology. Perhaps unsurprisingly, these are the products that were used for the 2001 events: vaporous hydrogen peroxide, and gaseous chlorine dioxide.

\subsubsection{Chlorine dioxide}

The following items were identified in SAIC (2005) as disadvantages for the use of chlorine dioxide in remediation.

1. The gas is unstable and must be constantly replaced to attain the target concentration for the required time.

2. The gas must be generated onsite, and the equipment required to do this can be significant.

3. The killing efficiency decreases significantly at relative humidity levels below 70 percent. Maintenance of humidity is critical.

4. A large volume of liquid waste is generated.

5. Some reports from field fumigations suggest that some collateral damage may occur to the surfaces of machinery and electrical systems, resulting from condensation.

The following items were identified in SAIC (2005) as advantages for the use of chlorine dioxide in remediation.

1. Chlorine dioxide gas is known to be effective: it is well documented as a disinfectant for spores, vegetative bacteria and viruses.

2. Rapid natural breakdown of $\mathrm{ClO} 2$ eases its removal after application: liquid wastes generated are low toxicity.

3. The gas is very soluble and stable in water.

4. The gas is effective on porous and non-porous surfaces and reaches all regions within an enclosure except for the hardest to reach, isolated areas (e.g., closed employee lockers). 
5. The gas can be commercially generated by several methods.

6. The gas odor can be detected by humans at a concentration ( $0.1 \mathrm{ppm})$ equal to the permissible exposure limit (PEL).

\subsubsection{Hydrogen Peroxide Vapor}

The only item identified in SAIC (2005) as a disadvantage for the use of hydrogen peroxide vapor in remediation is that it is highly reactive and "can break down upon contact with certain materials such as galvanized steel and porous surfaces such as paper and unpainted cinderblock." This can greatly reduce its effectiveness. Also, VHP is very toxic, although since it is also an irritant at low concentrations it would be unlikely that someone would be unknowingly exposed to a dangerous dose.

The main advantages of hydrogen peroxide vapor are that it is a routinely used technology that is readily available at a scale useable for buildings, and that its end products are water and oxygen.

\subsubsection{Recommendations}

For whole-building fumigation, chlorine dioxide gas and hydrogen peroxide vapor remain the most attractive options. Both can be handled safely, are known to be effective against anthrax, and are readily available. Chlorine dioxide apparently has an edge in effectiveness, because hydrogen peroxide breaks down in the presence of galvanized steel (including ventilation ducts). However, hydrogen peroxide has an edge in convenience, because it is easier to generate and to work with, and because it does not create a substantial volume of liquid waste.

For surface treatment, quite a few products are available, ranging from dilute common bleach to specially formulated foams. The foams may have an advantage in certain circumstances, such as vertical surfaces for which liquids do not have enough contact time, but liquid chlorine dioxide is inexpensive, effective, and easy to use, so it may be the most attractive option. See the EPA report "Compilation of Available Data on Building Decontamination Alternatives" (SAIC, 2005) for information about specific agents.

For decontamination of critical documents, gamma radiation should be considered as an alternative to paraformaldehyde or other agents, even for objects other than mail. Helfinstine et al. ( 2005) show reduction in B. atrophaeus by $10^{8}$ from $16 \mathrm{kGy}$ dose of gamma radiation, for spores at the bottom of a $5.5 \mathrm{~cm}$ stack of envelopes. US Postal Service uses 50 to $100 \mathrm{kGy}$, which appears to provide a margin of safety even for larger documents such as books or files. Especially since USPS is already equipped to irradiate large quantities of material, this option might be attractive.

\subsection{Acknowledgements}

Funding for this report was provided by the Department of Homeland Security. 
Marc Traeger of the Centers for Disease Control provided data and maps of anthrax measurements in the AMI building. Ken Martinez, also of the Centers for Disease Control, provided contact information and advice. Mark Durno of the U.S. Environmental Protection Agency provided several useful reports concerning the Capitol Hill anthrax response. Tom Sgroi of the State Department sent reports about anthrax testing in the Sterling Mail Annex. Sean McKenna sent data detailing the spatial distribution of particles deposited in experiments performed by Sandia National Laboratories. 


\section{Bibliography}

[1] Agranovski IE, Pyankov OV, and Altman IS. Bioaerosol contamination of ambient air as a result of opening envelopes containing microbial materials. Aerosol Science and Technology 39:1048-1055, 2005.

[2] Barth E, Rupert R, Stroud F, Rice E, and Potoka B. Environmental response to intentional dissemination of Bacillus Anthracis spores in the United States - 2001. Remediation, 99-111, 2003.

[3] Brown GS, Betty RG, Brockmann JE, Lucero DA, Souza CA, Walsh KS, Bougher RM, Tezak M, and Wilson MC, Evaluation of Wipe Surface Sample Collection Method for Bacillus Spores from Non-Porous Surfaces, Applied and Environmental Microbiology, 73:706-710, 2007.

[4] Burrows W. Textbook of microbiology. Philadephia, PA: W.A. Saunders Publishing (1973).

[5] Buttner MP, Cruz-Perez P, and Stetzenbach LD. Enhanced detection of surfaceassociated bacteria in indoor environments by PCR. Applied and Environmental Microbiology 67:2564-2570, 2001.

[6] Buttner MP, Cruz P, Stetzenbach LD, Klima-Comba AK, Stevens VL, and Cronin TD. Determination of the efficacy of two building decontamination strategies by surface sampling with culture and quantitative PCR analysis. Applied and Environmental Microbiology, 70:4740-4747, 2004a.

[7] Buttner MP, Cruz P, Stetzenbach LD, Klima-Comba AK, Stevens VL, and Emanuel PA. Evaluation of the biological Sampling Kit (BiSKit) for large-area surface sampling. Applied and Environmental Microbiology, 70:7040-7045, 2004b.

[8] Buttner MP, Cruz-Perez P, Stetzenbach LD, Garrett PJ, and Lutke AE. Measurement of airborne fungal spore dispersal from three types of flooring materials, Aerobiologia 18(1): 1-11, 2002.

[9] Buxton, B. Memo to Sineta Wooten, U.S. Environmental Protection Agency, from Measurement and Data Analysis Sciences. February 8, 2005.

[10] CDC "Anthrax Q \& A: Laboratory Testing," http://www.bt.cdc.gov/agent/anthrax/faq/labtesting.asp, December 2007. 
[11] Dahlgren CM, Buchanan LM, Decker HM, et al. Bacillus anthracis aerosols in goat hair processing mills. American Journal of Hygeine, 72:24-31, 1960.

[12] Dull PM, Wilson KE, Kournikakis B, Whitney EAS, Boulet CA, Ho JYW, Ogston J, Spence MR, McKenzie MM, Phelan MA, Popovic T, and Ashford D. Bacillus anthracis aerosolization associated with a contaminated mail sorting machine. Emerging Infectious Diseases 8:1044-1047, 2002.

[13] Dun S, Report on the 2006 Workshop on Decontamination, Cleanup, and Associated Issues for Sites Contaminated with Chemical, Biological, or Radiological Materials, Prepared for US EPA, EPA/600/R-06/121, January 2007.

[14] EPA 2002. Federal on-scene coordinator's report for the Capitol Hill site, Washington DC. United States Environmental Protection Agency, Region 3, Philadelphia, Pennsylvania, 2002.

[15] Farnsworth JE, Goyal SM, Kim SW, Kuehn TH, Raynor PC, Ramakrishnan MA, Anantharaman S, and Tang W. Development of a method for bacteria and virus recovery from heating, ventilation, and air conditioning (HVAC) filters. Journal of Environmental Monitoring 8:1006-1013, 2006.

[16] Fennelly KP, Davidow AL, Miller SL, Connell N, and Ellner JJ. Airborne infection with Bacillus anthracis-from Mills to Mail. Emerging Infectious Diseases 10:9961001, 2004.

[17] Fitch JP, Raber E, and Imbro DR. Technology challenges in responding to biological or chemical attacks in the civilian sector. Science 302:1350-1354, 2003.

[18] Gilbert RO, Hassig NL, Wilson JE, Nuffer LL, and Pulsipher BA. Technical documentation and verification for the buildings module in the Visual Sampling Plan (VSP) software. Pacific Northwest Laboratory PNNL-15202, 2005.

[19] Glassman NH. Discussion of industrial inhalation anthrax. Bacteriology Review 30:657-659, 1966.

[20] Griffith RO, Ramsey JL, Finley PD, Melton BJ, Brockmann JE, Lucero DA, McKenna SA, Peyton CE, Knowlton RG, Einfeld W, Ho P, Brown GS, and Tucker MD. Joint Sandia/NIOSH exercise on aerosol contamination using the BROOM tool. Sandia National Laboratories, Albuquerque, New Mexico. Document number SAND2006-3784, 2006.

[21] Helfinstine SL, Vargas-Aburto C, Uribe RM, and Woolverton CJ. Inactivation of Bacillus Endospores in Envelopes by Electron Beam Irradiation, Applied and Environmental Microbiology, 71:70297032, Nov. 2005.

[22] Heller MB, Bunning ML, France MEB, Niemeyer DM, Peruski L, Naimi T, Talboy PM, Murray PH, Pietz HW, Kornblum J, Oleszko W, Beatrice ST, Joint Microbiological Rapid Response Team, and New York City Anthrax Investigation Working Group. Laboratory response to anthrax bioterrorism, New York City, 2001. Emerging Infectious Diseases 8, October 2002. 
[23] Inglesby TV, OToole T, Henderson DA, Bartlett JG, Ascher MS, Eitzen E, Friedlander AM, Gerberding J, Hauer J, Hughes J, McDade J, Osterholm MT, Parker G, Perl TM, Russell PK, Tonat K, Anthrax as a biological weapon, 2002: Updated recommendations for management. Journal of the American Medical Association 287:2236-2252, 2002.

[24] Mitretek Systems, Review of Decontamination Technologies for Biological and Chemical Warfare Agents In Support of the U.S. Environmental Protection Agency's Safe Buildings Program. U.S. EPA. Draft, not released to the public. Washington, DC, March 2003.

[25] NRC (National Research Council). Reopening public facilities after a biological attack: A decision-making framework. Committee on Standards and Policies for Decontaminating Public Facilities Affected by Exposure to Harmful Biological Agents: How Clean is Safe? National Academies Press (2005).

[26] Peters CJ and Hartley DM. Anthrax inhalation and lethal human infection. Lancet, 359:710-711, 2002.

[27] Resnick IG, Martin DD, and Larsen LD. Evaluation of need for detection of surface biological agent contamination. Dugway Proving Ground publication DPG-FR-90$711,1990$.

[28] Rhodes, KA. Agencies need to validate sampling activities in order to increase confidence in negative results. Testimony before the Chairman, Subcommittee on National Security, Emerging Threats, and International Relations, House Committee on Government Reform, House of Representatives of the United States. Government Accounting Office document GAO-05-493T, April 2005.

[29] Rose L, Jensen B, Peterson A, Banerjee SN, and Arduino MJ. Swab materials and Bacillus anthracis spore recovery from nonporous surfaces. Emerging Infectious Diseases 10, June 2004.

[30] Sajo E, Zhu H, and Courtney JC. Spatial distribution of indoor aerosol deposition under accidental release conditions. Health Physics 83:871-883, 2002.

[31] Sanderson WT, Hein MJ, Taylor L, Curwin BD, Kinnes GM, Seitz TA, Popovic T, Holmes HT, Kellum ME, McAllister SK, Whaley DN, Tupin EA, Walker T, Freed JA, Small DS, Klusaritz B, and Bridges JH. Surface sampling methods for Bacillus anthracis spore contamination. Emerging Infectious Diseases 8:1145-1151, 2002.

[32] Sanderson WT, Stoddard RR, Echt AS, Piacitelli CA, Kim D, Horan J, Davies MM, McCleery RE, Muller P, Schnorr TM, Ward EM, and Hales TR. Bacillus anthracis contamination and inhalational anthrax in a mail processing and distribution center. Journal of Applied Microbiology 96:1048-1056, 2004.

[33] Sandia (2006). Sandia tool speeds up environmental cleanup, reopening of contaminated facility: BROOM software now available to potential licensing partners. Press Release, Sandia National Laboratories. June 7, 2006. 
[34] SAIC: Science Applications International Corporation. Compilation of Available Data on Building Decontamination Alternatives. Prepared for US EPA, EPA/600/R-05/036, March 2005.

[35] Sharp RJ and Roberts AG, Anthrax: the challenges for decontamination. Journal of Chemical Technology and Biotechnology 81:1612-1625, 2006.

[36] Sippola MR, and Nazaroff WW. Modeling particle loss in ventilation ducts. Atmospheric Environment 37:5597-5609, 2003.

[37] Skolnick EB and Hamilton RG. "Legacy" science suggests improved surface-testing practices for detection of dispersed bioagents (e.g. Bacillus anthracis spores) in bioterrorism response. Proceedings of the 20th Annual Environmental Monitoring Conference, 2004.

[38] Snyder JW, Shapiro DS, Gilchrist MJ, Weyant RS, Popovic T, Ezzell JW, and Morse SA. Basic diagnostic testing protocols for Level A laboratories for the presumptive identification of Bacillus anthracis. Centers for Disease Control, 2002.

[39] Spotts Whitney EA, Beatty ME, Taylor TH Jr., Weyant R, Sobel J, Arduino MJ, and Ashford DA. Inactivation of Bacillus anthracis spores. Emerging Infectious Diseases 9:623-627.

[40] Stuart AL and Wilkening DA. Degradation of biological weapons agents in the environment: Implications for terrorism response, Environmental Science and Technology 39 (8): 2736-2743, April 152005

[41] Teshale EH, Painter J, Burr GA, Mead P, Wright SV, Cseh LF, Zabrocki R, Collins R, Kelley KA, Hadler JL, Swerdlow DL, and members of the Connecticut Anthrax Response Team. Environmental sampling for spores of Bacillus anthracis. Emerging Infectious Diseases 8:1083-1087, 2002.

[42] Traeger MS, Wiersma ST, Rosenstein NE, Malecki JM, Shepard CW, Raghunathan PL. First case of bioterrorism-related inhalational anthrax in the United States, Palm Beach, Florida, 2001. Emerging Infectious Diseases 8:1029-1034, October 2002 .

[43] USDA 2003. National Animal Health Emergency Management System Guidelines, Operational Guidelines: Cleaning and Disinfection. U.S Department of Agriculture, November 2003.

[44] U. S. Postal Service Emergency Preparedness Plan for Protecting Postal Employees and Postal Customers From Exposure to Biohazardous Material and for Ensuring Mail Security Against Bioterror Attacks, U.S. Postal Service, 2002.

[45] Vogt BM and Sorensen JH, How Clean is Safe? Improving the Effectiveness of Decontamination of Structures and People Following Chemical and Biological Incidents, ORNL/TM-2002/178, Oak Ridge National Laboratory, 2002. 
[46] Webb GF, and Blaser MJ. Mailborne transmission of anthrax: modeling and implications. Proceedings of the National Academy of Science of the U.S.A. 99:7027-7032, 2002.

[47] Wein LM, Liu Y, and Leighton TJ, HEPA/Vaccine plan for indoor anthrax remediation. Emerging Infectious Diseases 11:69-76, 2005.

[48] Weis CP, Intrepido AJ, Miller AK, Cowin PG, Durno MA, Gebhardt JS, and Bull R. Secondary aerosolization of viable Bacillus anthracis spores in a contaminated U.S. Senate office. Journal of the American Medical Association 288: 2853-2858, 2007.

[49] Wilkening DA, Sverdlosk Revisited: Modeling Human Inhalation Anthrax. Proceedings of the National Academy of Sciences of the United States of America, 103:7589-7594, 2006. 